\title{
Design, synthesis and activity of thio-linked arabinofuranosyl disaccharides against mycobacterial tuberculosis (MTB) and Mycobacterium avium complex (MAC)
}

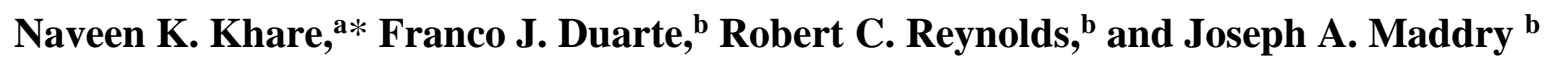 \\ ${ }^{a}$ Department of Chemistry, University of Lucknow, Lucknow 226 007, India \\ ${ }^{b}$ Southern Research Institute, 2000 Ninth Avenue South, P.O. Box 55305, \\ Birmingham, AL 35255, USA \\ E-mail: nkhare58@gmail.com
}

Dedicated to Professor Richard R. Schmidt on the occasion of his $\mathbf{7 8}^{\text {th }}$ anniversary

\begin{abstract}
We report the chemical synthesis of a series of disaccharides of arabinofuranose with a glycosidic sulfur linker as mimics of the acceptor for arabinofuranosyltransferases with and without using any activator to avoid any complex reactions. These analogs were tested for in vitro activity against MTB strain H37Ra and 3 MAC clinical isolates. MICs were determined using a colorimetric microdilution broth assay. Bactericidal activity was studied with kill curves over a period of seven days. Intracellular activity against MTB H37Ra was determined in the Mono Mac 6 (MM6) human monocytic cell line.
\end{abstract}

Keywords: Arabinofuranosyl transferases, inhibitors, arabinofuranose, thio linked disaccharides, mycobacteria, bacterial cell walls

\section{Introduction}

The resurgence of tuberculosis in developed nations as well as the appearance of multiple drug resistant forms of the disease throughout the world has raised the concern that this disease may resurface as serious public health problem ${ }^{1-3}$ and this attracted renewed attention in identifying the potent antimycobacterial agents. Also, the effective chemotherapy of persons with AIDS who are infected with Mycobacterium avium can be difficult due to the inherent resistance of this organism. ${ }^{4}$ All Mycobacterium species share a characteristic cell wall, thicker than in many other bacteria, which is hydrophobic, waxy, and rich in mycolic acids/mycolates. The cell wall consists of the hydrophobic mycolate layer and a peptidoglycan layer held together by a polysaccharide, arabinogalactan. The cell wall makes a substantial contribution to the hardiness 
of this genus. The biosynthetic pathways of cell wall components are potential targets for new drugs for tuberculosis. Much of the pathogenicity of M. tuberculosis results from its unique and complex cell envelope. The major components of this mycobacterial envelope are the mycolyl arabinogalactanpeptidoglycan complexes (mAGPs) and the lipoarabinomannan (LAM)associated lipoglycans. The biosynthetic pathways of cell wall components are potential targets for new drugs for tuberculosis. The recent advances in the characterization of mycobacteria cell walls have led to the identification of a vast array of highly unique biochemical targets that could lead to a new generation of potent and selective anti tubercular agents. ${ }^{5}$ Arabinogalactan, a major and essential component of the cell wall, is an attractive target for drug development as neither D-arabinofuranose nor D-galactofuranose, the monomers composing arabinogalactan, are found in mammalian cells. ${ }^{6}$ The effectiveness of antituberculosis drug, Ethambutol, ${ }^{7}$ which inhibits the synthesis of arabinan, ${ }^{8}$ a cell envelope component, illustrates the importance of this structure to the survival of the organism. Since several natural and synthetic arabinosyl glycosides are known to be substrate of mycobacterial arabinosyl transferase, ${ }^{9}$ we hypothesized that simple arabinosyl disaccharide incorporating a sulfur atom that could potentially chelate the putative cation might function as specific inhibitors.

The inhibitors of mycobacterial arabinofuranosyl transferases could be the ideal synthetic targets as neither D-arabinofuranose nor D-galactofuranose, the monomer composing arabinogalactan are found in mammalian cells. Recently, several oligosaccharides with sulfur in the glycosidic linkage are investigated as potential inhibitors of glycosyltransferases. Such compounds should also reduce hydrophilicity and enhance hydrolytic and enzymatic stability.

We have initiated a program to synthesize a series of disaccharides with sulfur linkers as mimics of the acceptor for arabinofuranosyl transferase. Such compounds should also reduce hydrophilicity and enhance hydrolytic and enzymatic stability.

Herein we report for the first time the synthesis, characterization and biological evaluation of several regioselectively protected D-arabinofuranosyl-(1,5)-D-arabinofuranosides with thiolinked octyl groups. An octyl group has been shown to be suitable for studies of mycobacterial arabinosyltransferases and other glycosyltransferases. ${ }^{6 \mathrm{~b}, 7 \mathrm{~b}, 9 \mathrm{~b}, 10}$

\section{Results and Discussion}

Thio-linked oligosaccharides involving hexoses have been synthesized earlier by a variety of procedures, (a) a $S_{N} 2$ type reaction involving the reaction of a thiolate anion on a glycosyl halide, ${ }^{11}$ and (b) the displacement of a leaving group by a 1-thioglycose. ${ }^{12}$ Initially our approach was based on the former procedure to yield thio-linked arabinofuranoside, $\mathbf{9 a}, \mathbf{b}, \mathbf{c}$ but the latter procedure was applied in a modified displacement reaction for the synthesis of the rest of the disaccharides to avoid using the toxic mercury salts as an activator and also to avoid any complex side reactions. An attempt was also made to couple the 2,3,5-tri- $O$-benzoyl arabinofuranosyl trichloroacetimidate ${ }^{13}$ with octyl-2,3-di- $O$-acetyl-5-thio- $\alpha$-D-arabinofuranoside ${ }^{13}$ using 
the promoters $\mathrm{BF}_{3} \mathrm{OEt}_{2}$ or $\mathrm{TESOTfl}^{14}$ which resulted in an inseparable mixture of symmetric $\alpha, 1-1, O$-linked arabinofuranoside from donor, disulfide from acceptor and traces of the desired disaccharide (Scheme 1).

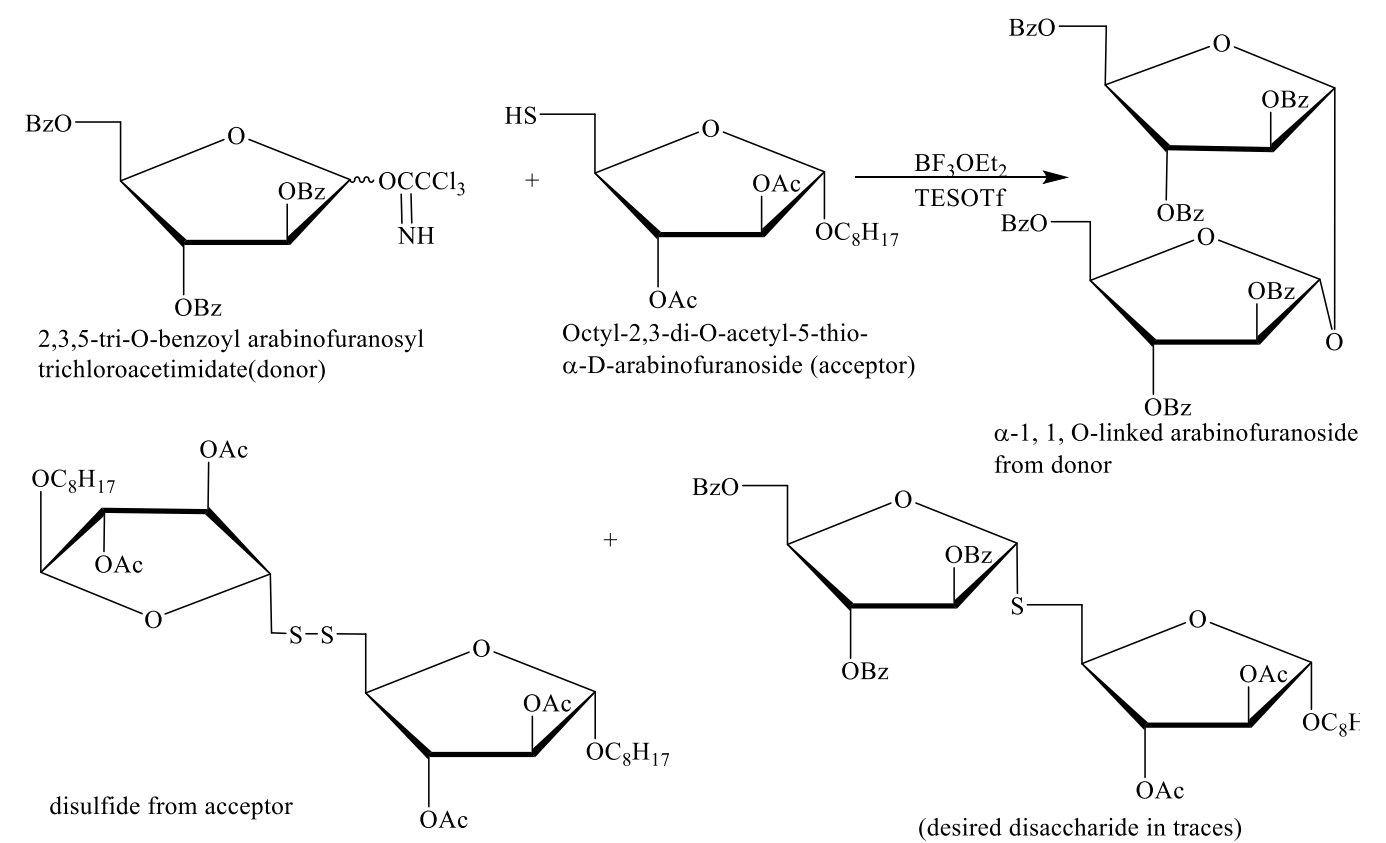

Scheme 1. Attempted synthesis of thio-linked disaccharides.

The octyl (3a), 1-methylpropyl (sec-butyl) (3b) and 3-phenylpropyl (3c) arabinosides were obtained separately from methyl-2,3,5-tri- $O$-benzoyl- $\alpha$-D-arabinofuranoside ${ }^{15}$ (1) through the glycosyl bromide (2) ${ }^{16}$ with $\mathrm{SnCl}_{4}{ }^{17}$ (Scheme 2). Another two-step protection/deprotection sequence yielded 6 which on reaction with potassium thioacetate gave 7 . Compound 7 upon treatment with sodium methoxide and coupling with $\mathbf{2}$ using mercuric cyanide as an activator afforded $\mathbf{8}$ in good yield. The target compounds $\mathbf{9 a - c}$ were obtained after debenzoylation of $\mathbf{8}$.

Compound $\mathbf{1 0}$ was obtained on treating $\mathbf{1}^{15}$ with thiolacetic acid in boron trifluoride etherate which upon reaction with sodium methoxide gave 11 (Scheme 3). The coupling of 11 and 12 (obtained from $\mathbf{6 a}$ on refluxing with sodium iodide in 2-butanone) on displacing the iodide of $\mathbf{1 2}$ without using any activator and followed by acetylation did not yield the expected $\alpha$-linked thio disaccharide but $\beta$-linked $\operatorname{ara} p(\mathrm{~S}, 1-5)$ araf $(\mathbf{1 3})$ and $\operatorname{ara} f(\mathrm{~S}, 1-5)$ ara $f(\mathbf{1 4})$. 


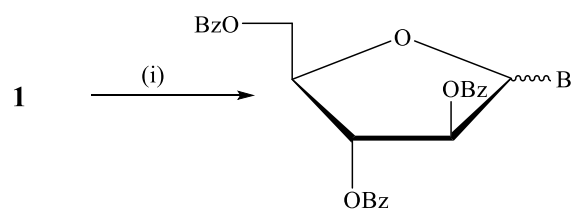

2

7

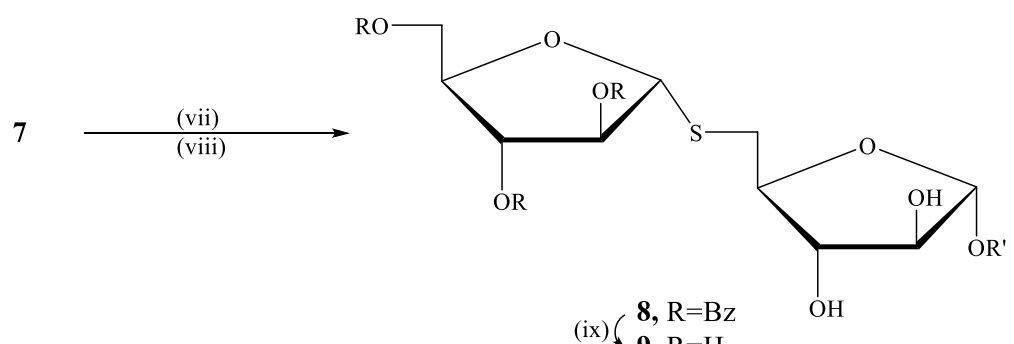

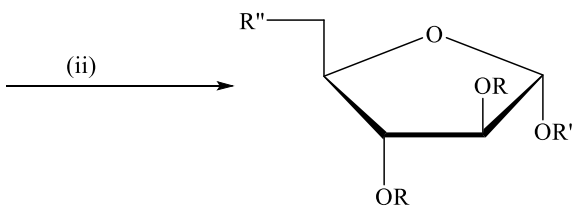

(iii) $3, \mathrm{R}=\mathrm{Bz}, \mathrm{R}=\mathrm{OBz}$ (iv) $4, \mathrm{R}=\mathrm{H}, \mathrm{R}=\mathrm{OH}$

$5, \mathrm{R}=\mathrm{H}, \mathrm{R}=\mathrm{OTs}$

(v) $6, \mathrm{R}=\mathrm{Ac}, \mathrm{R}^{\prime \prime}=\mathrm{OTs}$

(vi) $7, \mathrm{R}=\mathrm{Ac}, \mathrm{R}^{\prime \prime}=\mathrm{SAc}$

a, $\mathrm{R}^{\prime}=\mathrm{C}_{8} \mathrm{H}_{17} ; \mathrm{b}, \mathrm{R}^{\prime}=\mathrm{CH}\left(\mathrm{CH}_{3}\right) \mathrm{CH}_{2} \mathrm{CH}_{3} ; \mathrm{c}, \mathrm{R}^{\prime}=\mathrm{CH}_{2} \mathrm{CH}_{2} \mathrm{CH}_{2} \mathrm{C}_{6} \mathrm{H}_{5}$

Reagents \& Conditions- (i) $30 \% \mathrm{HBr}$ in glacial $\mathrm{AcOH}, \mathrm{RT}, 1 \mathrm{~h}$; (ii) $\mathrm{R}^{\prime} \mathrm{OH}, \mathrm{SnCl}_{4}, \mathrm{RT}, 15 \mathrm{~h}$; (iii) $\mathrm{NH}_{3}$, $\mathrm{MeOH}, \mathrm{RT}, 22 \mathrm{~h}$; (iv) $\mathrm{C}_{5} \mathrm{H}_{5} \mathrm{~N}$, TsCl, $0^{\circ}-\mathrm{RT}, 24 \mathrm{~h}$; (v) $\mathrm{C}_{5} \mathrm{H}_{5} \mathrm{~N}, \mathrm{Ac}_{2} \mathrm{O}, \mathrm{RT}, 15 \mathrm{~h}$; (vi) KSAc, RT, 26h; (vii)

Scheme 2. Synthesis of the thio-linked disaccharides 9a-c.
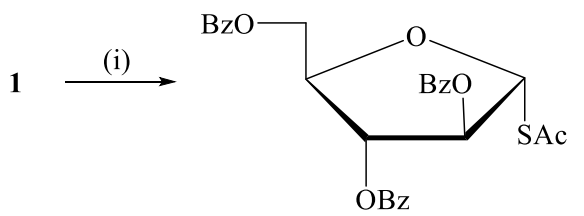

10

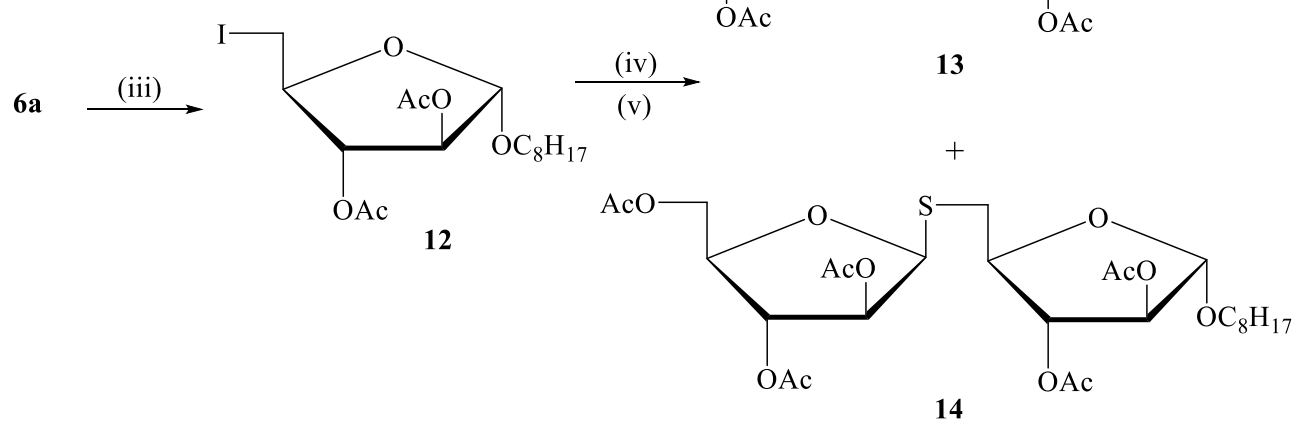

Reagents \& Conditions: (i) $\mathrm{AcSH}, \mathrm{BF}_{3} \mathrm{OEt}_{2}, \mathrm{RT}, 18 \mathrm{~h}$; (ii) $\mathrm{MeONa}, 0^{\circ}$ to $\mathrm{RT}, 2 \mathrm{~h}$; (iii) NaI, 2-butanone, $85^{\circ}, 18 \mathrm{~h}$; (iv) 11, DMF, RT, $18 \mathrm{~h}$; (v) $\mathrm{C}_{5} \mathrm{H}_{5} \mathrm{~N}, \mathrm{Ac}_{2} \mathrm{O}, \mathrm{RT}, 18 \mathrm{~h}$.

Scheme 3. Synthesis of the thio-linked disaccharides 13 and $\mathbf{1 4 .}$ 
To obtain the desired $\alpha$-linked disaccharide, compound 18 synthesized from $\mathbf{1}^{15}$ after three steps of protection/deprotection techniques, on coupling with tosyl derivative(6a) yielded only the $\alpha$-linked thiodisaccharide 19 (Scheme 4).

Similarly, the $\alpha$-linked thiodisaccharide $\mathbf{2 6}$ was made from $\mathbf{1 5}^{18}$ as follows. Compound $\mathbf{1 5}^{18}$ upon methylation gave $\mathbf{2 0}$ followed by acetolysis with thiol acetic acid yielded $\beta$ and $\alpha$ thioacetates 21 and 22, respectively. After chromatographic separation, both the thiosodium derivatives $\mathbf{2 3}$ and $\mathbf{2 4}$ were reacted separately with $\mathbf{6 a}$, in absence of any activator, gave only $\alpha$ linked thio disaccharide 25 irrespective of the anomeric configuration of starting thioacetates 21 and 22, which on deacetylation gave 26 (Scheme 5).

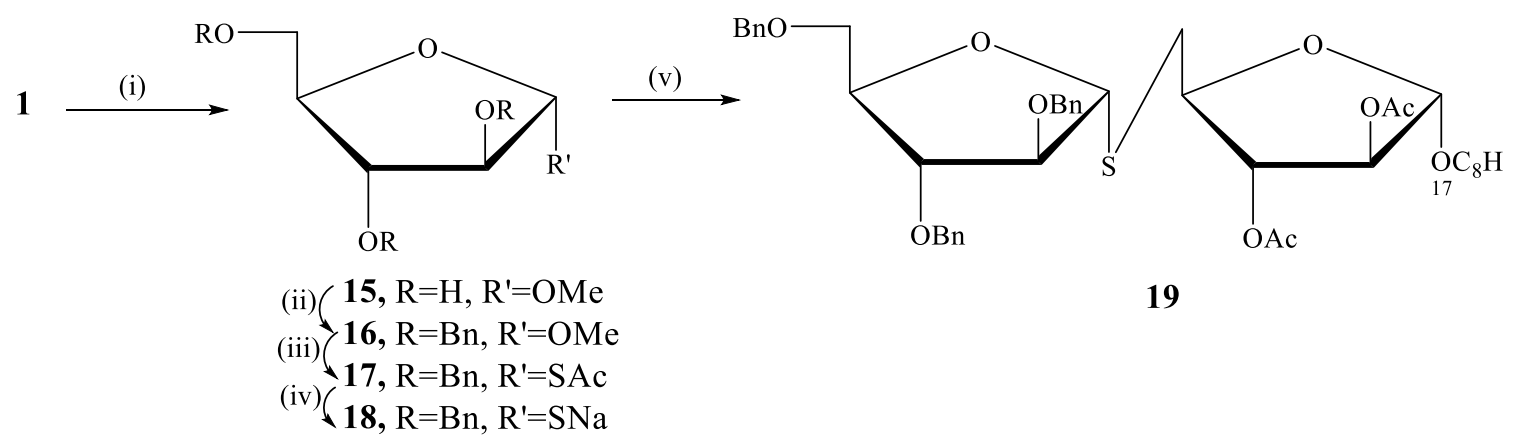

Reagents \& Conditions- (i) $\mathrm{NH}_{3}, \mathrm{MeOH}, \mathrm{RT}, 18 \mathrm{~h}$; (ii) $\mathrm{NaH}, \mathrm{BnBr}, 0^{\circ}$ to RT, $18 \mathrm{~h}$; (iii) AcSH, $\mathrm{BF}_{3} \mathrm{OEt}_{2}$, RT, 18h; (iv ) $\mathrm{NH}_{3}, \mathrm{MeOH}, \mathrm{RT}, 18 \mathrm{~h}$; (v) 6a, DMF, RT, $18 \mathrm{~h}$.

Scheme 4. Synthesis of the thio-linked disaccharide 19.
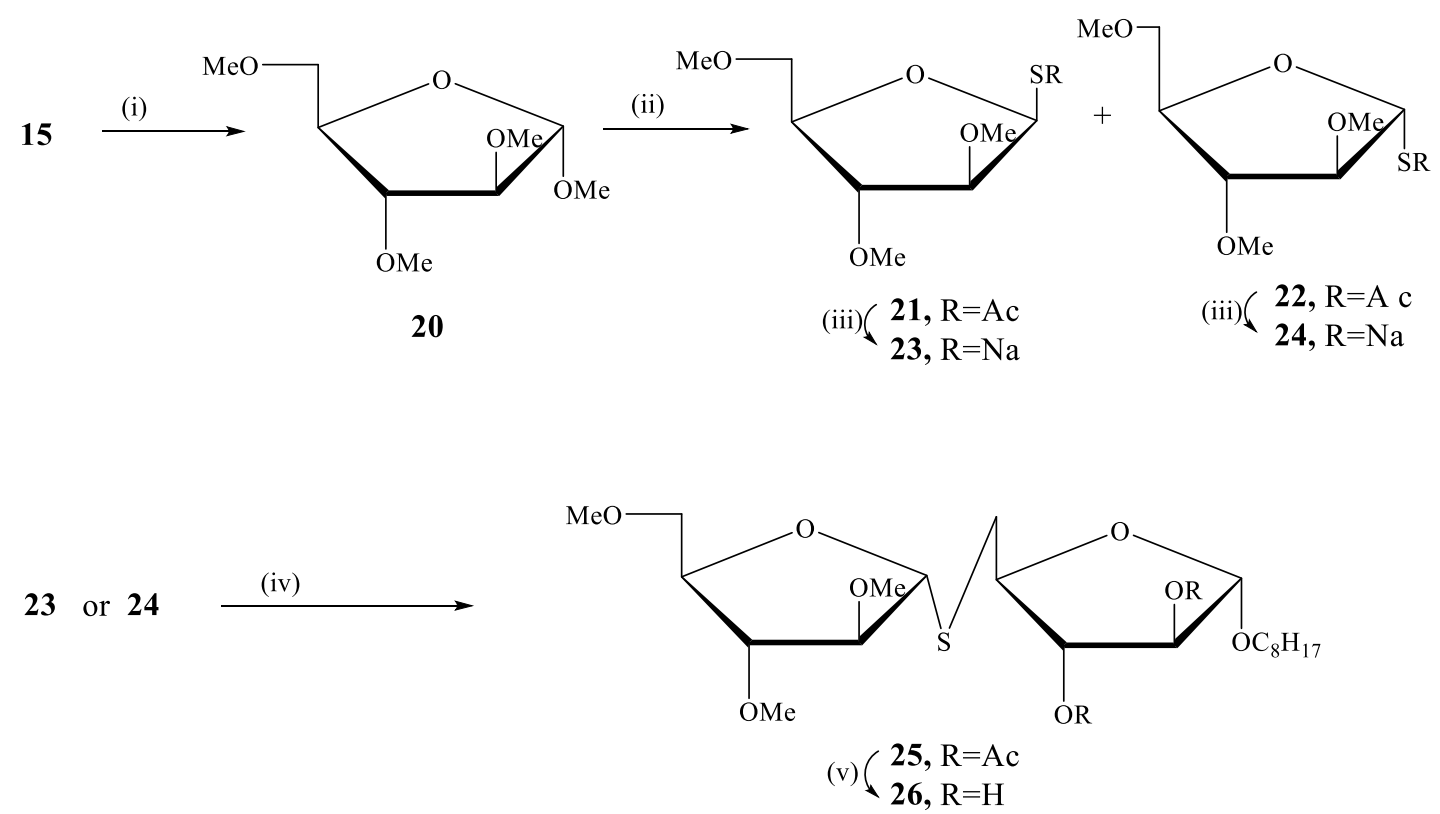

Reagents \& Conditions- (i) NaH, MeI, $0^{\circ}$ to RT, $18 \mathrm{~h}$; (ii) $\mathrm{AcSH}, \mathrm{BF}_{3} \mathrm{OEt}_{2}, \mathrm{RT}, 18 \mathrm{~h}$; (iii) $\mathrm{MeONa}, 0^{\circ}$ to RT, 2h; (iv) 6a, DMF, RT, 48h; (v) $\mathrm{NH}_{3} / \mathrm{MeOH}$, RT, $18 \mathrm{~h}$.

Scheme 5. Synthesis of the thio-linked disaccharide 26. 
Compound 34 was obtained from $15^{18}$ on making the 5-O-TBDPS-derivative ${ }^{19}$ (27) followed by alkylation to get 2,3-di- $O$-methoxybenzyl, 28, which upon desilylation (29), benzylation (30), demethoxybenzylation (31) and acetylation gave 32. Compound 32 on reaction with thiolacetic acid in boron trifluoride etherate yielded $\alpha$-thioacetate $\mathbf{3 3}$ which ultimately gave the thiosodium 34 with sodium methoxide. Compound 34 was condensed with $6 \mathbf{a}$ followed by acetylation resulted in thiodisaccharides $\mathbf{3 5}$ and $\mathbf{3 6}$ (Scheme 6).
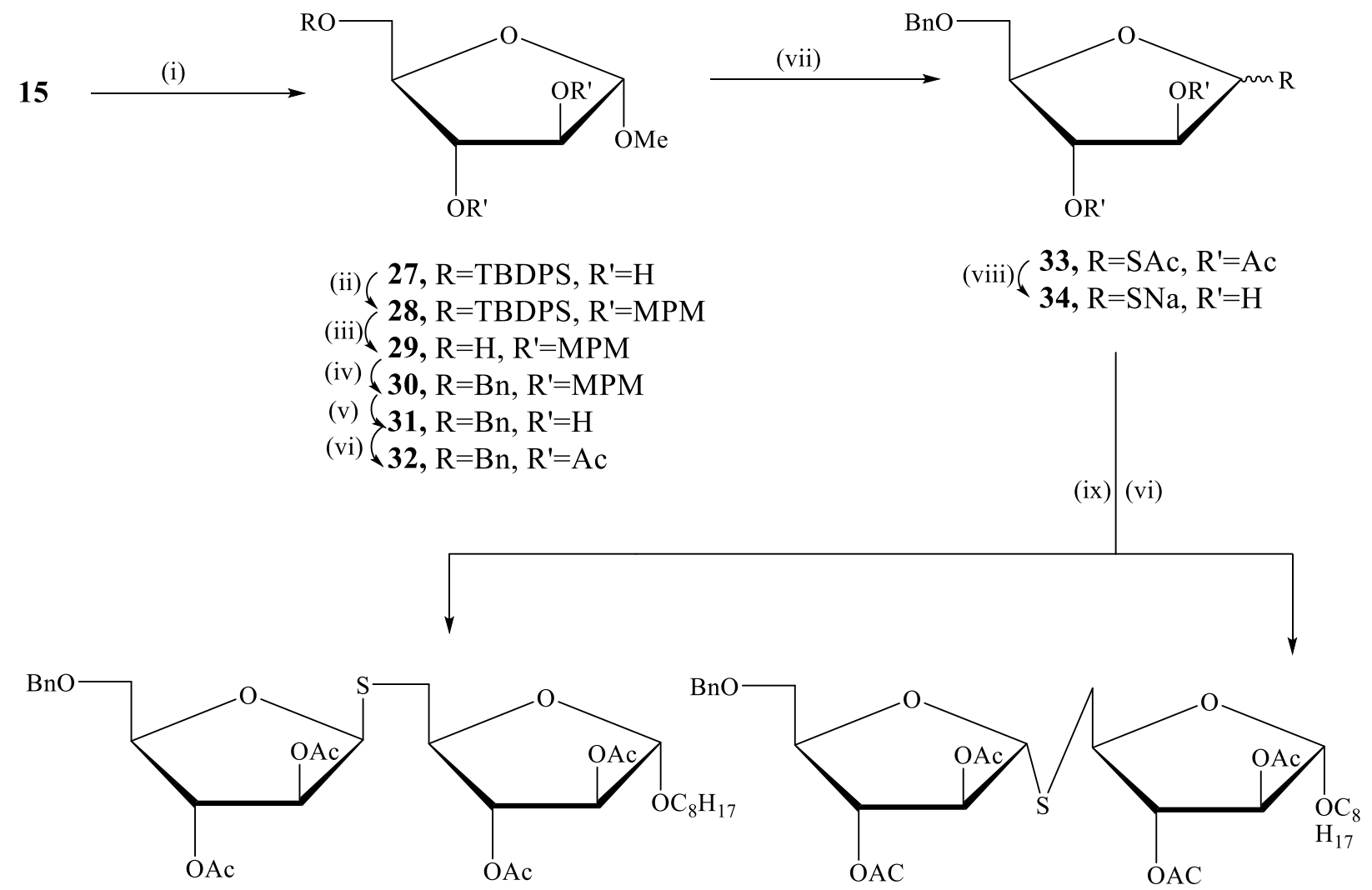

35

36

Reagents \& Conditions- (i) TBDPSCl, Imidazole, RT, 3h; (ii) NaH, MPMCl, TBABr, $0^{\circ}$ to RT, $18 \mathrm{~h}$; (iii) TBAF, $0^{\circ}$ to RT, $18 \mathrm{~h}$; (iv) $\mathrm{NaH}, \mathrm{BnBr}, 0^{\circ}$ to RT, $18 \mathrm{~h}$; (v) CAN, RT, 4h; (vi) $\mathrm{C}_{5} \mathrm{H}_{5} \mathrm{~N}, \mathrm{Ac}_{2} \mathrm{O}, \mathrm{RT}$, $18 \mathrm{~h}$; (vii) $\mathrm{AcSH}, \mathrm{BF}_{3} \mathrm{OEt}_{2}$, RT, $18 \mathrm{~h}$; (viii) MeONa, $0^{\circ}$ to RT, 2h; (ix) 6a, DMF, RT, 48h.

Scheme 6. Synthesis of the thio-linked disaccharides $\mathbf{3 5}$ and $\mathbf{3 6 .}$

Likewise, 5-deoxyarabinose 39 was obtained from $15^{18}$ on tosylation $(37)$, reduction with lithium aluminium hydride (38), and acetylation. Compound $\mathbf{3 9}$ was converted into $\alpha$-thioacetate 40 as reported earlier and coupled with 6a through 41 to give $5^{\prime}$-deoxythiodisaccharides $\mathbf{4 2}$ and 43 on acetylating the residue (Scheme 7). 


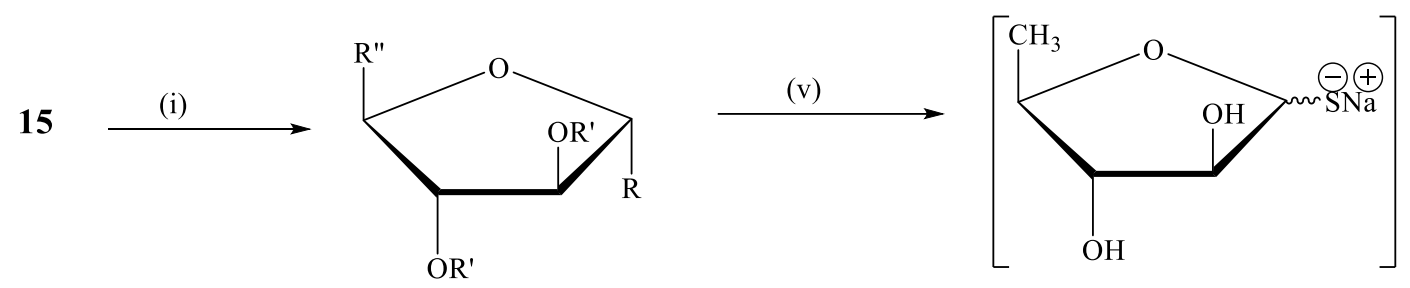

(ii) $37, \mathrm{R}=\mathrm{OMe}, \mathrm{R}^{\prime}=\mathrm{H}, \mathrm{R}^{\prime \prime}=\mathrm{CH}_{2} \mathrm{OTs}$

41

(iii) 38, $\mathrm{R}=\mathrm{OMe}, \mathrm{R}^{\prime}=\mathrm{H}, \mathrm{R}^{\prime \prime}=\mathrm{CH}_{3}$

(iv)

39, $\mathrm{R}=\mathrm{OMe}, \mathrm{R}=\mathrm{Ac}, \mathrm{R}=\mathrm{CH}_{3}$

40, $\mathrm{R}=\mathrm{SAc}, \mathrm{R}^{\prime}=\mathrm{Ac}, \mathrm{R}^{\prime \prime}=\mathrm{CH}_{3}$

(vi) (iii)

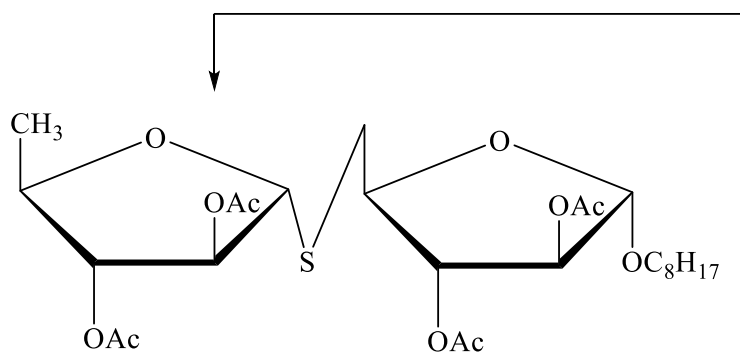

42

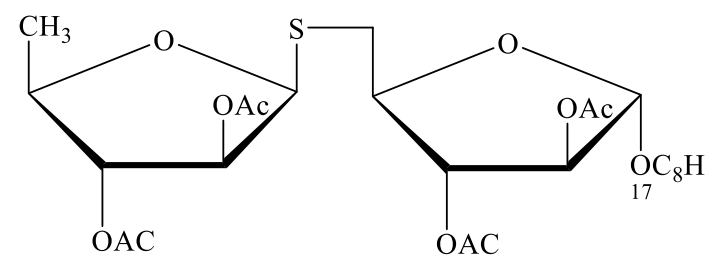

43

Reagents \& Conditions- (i) $\mathrm{C}_{5} \mathrm{H}_{5} \mathrm{~N}, \mathrm{TsCl}, 0^{\circ}$ to RT, 48h, (ii) LAH, RT, $18 \mathrm{~h}$, (iii) $\mathrm{C}_{5} \mathrm{H}_{5} \mathrm{~N}, \mathrm{Ac}_{2} \mathrm{O}, \mathrm{RT}, 18 \mathrm{~h}$; (iv) $\mathrm{AcSH}, \mathrm{BF}_{3} \mathrm{OEt}_{2}, \mathrm{RT}, 18 \mathrm{~h}$; (v) MeONa, $0^{\circ}$ to RT, 2h; (vi) 6a, DMF, RT, $48 \mathrm{~h}$.

Scheme 7. Synthesis of the thio-linked disaccharides $\mathbf{4 2}$ and $\mathbf{4 3 .}$

Compound 37 was refluxed with sodium azide to yield $\mathbf{4 4}$ which upon acetylation gave $\mathbf{4 5}$. The $\alpha$-thioacetate $\mathbf{4 6}$ was obtained from $\mathbf{4 5}$ with thiol acetic acid which ultimately yielded $\mathbf{4 7}$ with sodium methoxide. The coupling of $\mathbf{4 7}$ and $6 \mathrm{a}$ gave the expected disaccharides 48 and 49 as octylglycosides after acetylating the residue. The deacetylation of isolated $\alpha$ - and $\beta$-linked thiodisaccharides 48 and 49 yielded 50 and 51 respectively. Compound 49 upon treatment with triphenylphosphine in water followed by deacetylation gave 5 '-acetamidothiodisaccharide $\mathbf{5 2}$ (Scheme 8). 
37
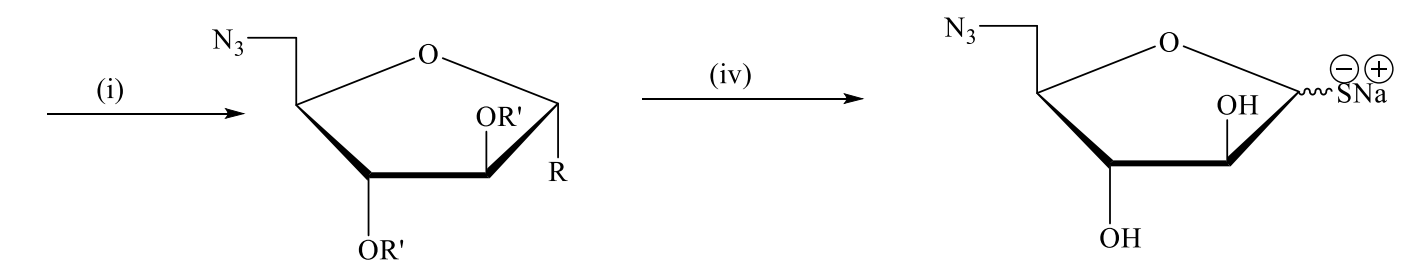

(ii) $\left(44, \mathrm{R}=\mathrm{OMe}, \mathrm{R}^{\prime}=\mathrm{H}\right.$

47

$$
\text { (iii) }\left(\begin{array}{l}
45, \mathrm{R}=\mathrm{OMe}, \mathrm{R}^{\prime}=\mathrm{Ac} \\
\mathbf{4 6}, \mathrm{R}=\mathrm{SAc}, \mathrm{R}^{\prime}=\mathrm{Ac}
\end{array}\right.
$$

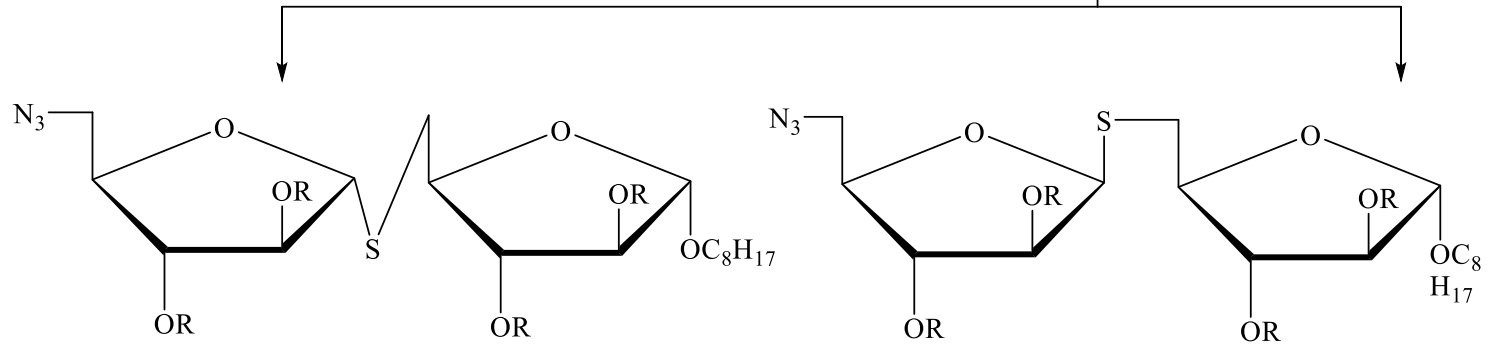

(vi) $\left(\begin{array}{l}\mathbf{4 8}, \mathrm{R}=\mathrm{Ac} \\ \mathbf{5 0}, \mathrm{R}=\mathrm{H}\end{array}\right.$

(vi) $\left(\begin{array}{l}49, \mathrm{R}=\mathrm{Ac} \\ 51, \mathrm{R}=\mathrm{H}\end{array}\right.$

49

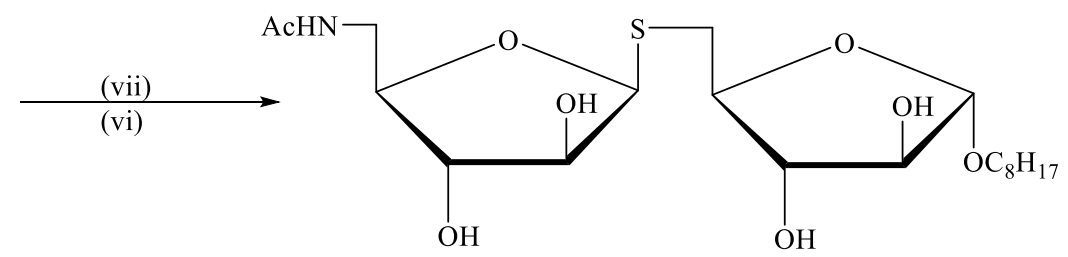

52

Reagents \& Conditions- (i) $\mathrm{NaN}_{3}, 100^{\circ}, 6 \mathrm{~h}$; (ii) $\mathrm{C}_{5} \mathrm{H}_{5} \mathrm{~N}, \mathrm{Ac}_{2} \mathrm{O}, \mathrm{RT}, 18 \mathrm{~h}$; (iii) AcSH, $\mathrm{BF}_{3} \mathrm{OEt}_{2}, \mathrm{RT}, 18 \mathrm{~h}$; (iv) MeONa, $0^{\circ}$ to RT, 2h; (v) 6a, DMF, RT, 48h; (vi) $\mathrm{NH}_{3} / \mathrm{MeOH}, \mathrm{RT}, 18 \mathrm{~h}$; (vii) $\mathrm{Ph}_{3} \mathrm{P}, \mathrm{H}_{2} \mathrm{O}, 65^{\circ}$, 5.5h.

Scheme 8. Synthesis of the thio-linked disaccharides 50-52. 


\section{Biological test data}

Table 1. Antimycobacterial activity of thio-linked disaccharides against Mycobacterium tuberculosis (MTB) and Mycobacterium avium Complex (MAC)

\begin{tabular}{ccccc}
\hline \multirow{4}{*}{ Test Compound } & \multicolumn{4}{c}{ MIC $(\mu \mathrm{g} / \mathrm{ml})$} \\
\cline { 2 - 5 } & MTB & MAC & MAC & MAC \\
& H37Ra & NJ 168 & NJ 211 & NJ 3404 \\
\hline $\mathbf{8}$ & 128 & $>128$ & $>128$ \\
$\mathbf{9}$ & 4 & $>128$ & 128 & $>12.8 \leq 128$ \\
$\mathbf{1 3}$ & $>128$ & $>128$ & $>128$ & $>128$ \\
$\mathbf{1 4}$ & $>128$ & $>128$ & $>128$ & $>128$ \\
$\mathbf{1 9}$ & $>128$ & $>128$ & $>128$ & $>128$ \\
$\mathbf{2 5}$ & $>128$ & $>128$ & $>128$ & $>128$ \\
$\mathbf{2 6}$ & $>128$ & $>128$ & $>128$ & $>128$ \\
$\mathbf{3 5}$ & $>128$ & $a$ & $>128$ & $a$ \\
$\mathbf{3 6}$ & $>128$ & $a$ & $>128$ & $a$ \\
$\mathbf{4 2}$ & $>128$ & $a$ & $>128$ & $a$ \\
$\mathbf{4 3}$ & $>128$ & $a$ & $>128$ & $a$ \\
$\mathbf{4 8}$ & $>128$ & $a$ & $>128$ & $a$ \\
$\mathbf{4 9}$ & $>128$ & $a$ & $>128$ & $a$ \\
$\mathbf{5 0}$ & 128 & $a$ & $>128$ & $a$ \\
$\mathbf{5 1}$ & 128 & $a$ & 128 & $a$ \\
$\mathbf{5 2}$ & $>128$ & $a$ & $>128$ & $a$ \\
Ethambutol (ETB) & $2-4$ & 8 & $4-8$ & 8 \\
\hline
\end{tabular}

$a$ - not determined.

Table 2. Activity of compound 9 against Mycobacterium tuberculosis H37Ra in infected human Mono Mac 6 macrophages a

\begin{tabular}{cccc}
\hline \multirow{2}{*}{$\mu \mathrm{g} / \mathrm{ml}$} & $\mu \mathrm{M}$ & \multicolumn{2}{c}{$\log _{10} \mathrm{CFU}$ (colony forming unit) } \\
\cline { 3 - 4 } & & Day 0 & Day 7 \\
\hline 0.0 & 0.0 & 4.94 & 5.41 \\
0.50 & 1.2 & - & 5.18 \\
4.0 & 9.8 & - & 5.18 \\
$32^{\mathrm{b}}$ & 78 & - & $4.34^{\mathrm{c}}$ \\
\hline
\end{tabular}

${ }^{\mathrm{a}}$ Rifamicin (positive control $: \log _{10}$ CFU @ $16 \mathrm{ng} / \mathrm{ml}=<4 .{ }^{\mathrm{b}}$ The macrophage viability was reduced by $39 \%$ of the control at this concentration. The $\mathrm{LD}_{50}$ for uninfected macrophages was $>48 \mu \mathrm{g} / \mathrm{ml}$. ${ }^{\mathrm{c}}$ Significant response $(\mathrm{P}<0.001)$. 
Compound 9 was active against $M$. tuberculosis with an MIC that was comparable to that observed with ethambutol. However, the thio-linked arabinofuranosyl disaccharide derivatives examined in this study tended to be less active against M. avium. Compound 9 was also bactericidal for $M$. tuberculosis and showed significant intracellular activity in a human macrophage cell line. However, the concentration of $\mathbf{9}$ that was active intracellularly also lowered the viability of the infected macrophages by $39 \%$. The possible contribution of this loss of macrophage viability to the decrease in viability of the intracellular M. tuberculosis is unclear. We have observed that when there is significant decrease in macrophage viability, $M$. tuberculosis viability can also be affected. Therefore, the CFU reduction may not have been due to the drug alone.

\section{Experimental Section}

General. The general procedures were same as reported earlier. ${ }^{20}$ The solvent used in NMR is $\mathrm{CDCl}_{3}$ unless otherwise stated. The structures of disaccharides were assigned on the basis of decoupling experiments in ${ }^{1} \mathrm{H}$ NMR.

Octyl- $\alpha$-D-arabinofuranoside (4a). To a solution of $\mathbf{2}^{16}(19.0 \mathrm{~g}, 36.1 \mathrm{mM})$ obtained from $\mathbf{1}^{15}$, in $\mathrm{CH}_{3} \mathrm{CN}(600 \mathrm{~mL})$ was added $n$-octanol $(10 \mathrm{~mL}, 63.5 \mathrm{mM})$ and $\mathrm{SnCl}_{4}(10 \mathrm{~mL}, 85.5 \mathrm{mM})$. The reaction mixture was stirred under argon at room temp. After $15 \mathrm{~h}$ the reaction mixture was diluted with sat $\mathrm{NaHCO}_{3}(127 \mathrm{~mL})$ and filtered through a celite pad. To the filtrate was added $\mathrm{CHCl}_{3}(260 \mathrm{~mL})$ and water $(260 \mathrm{~mL})$. The aqueous layer was extracted thrice with $\mathrm{CHCl}_{3}$. The combined organic layers were washed with aq. $\mathrm{NaHCO}_{3}$, water, dried $\left(\mathrm{MgSO}_{4}\right)$, filtered, and concentrated under reduced pressure to give crude yellow oil. Purification through a short column of silica gel (cyclohexane : EtOAc, $4: 1)$ gave 3a (14 g) as a yellow oil.

A solution of $3 \mathbf{a}(4.69 \mathrm{~g}, 8.17 \mathrm{mM})$ in dry $\mathrm{MeOH}(36 \mathrm{~mL})$ was treated with methanolic ammonia $(7 \mathrm{~N}, 36 \mathrm{~mL})$ under an atmosphere of argon. After $22 \mathrm{~h}$ at room temp the reaction mixture was concentrated under reduced pressure followed by column chromatography $\left(\mathrm{CHCl}_{3}: \mathrm{MeOH}, 9.5\right.$ : $0.5)$ gave 4a, $\left(5.30 \mathrm{~g}, 56 \%\right.$ for the last two steps). FAB-MS : $m / z$ 263(M+H) ${ }^{+} .{ }^{1} \mathrm{HNMR}: \delta 5.01$ $(1 \mathrm{H}, \mathrm{s}, \mathrm{H}-1), 4.16$ (1H, dd, J 4.2, $1.9 \mathrm{~Hz}, \mathrm{H}-4), 4.01$ (2H, m, H-2, H-3), 3.89 (1H, dd, J 11.8, 2.4 $\mathrm{Hz}, \mathrm{H}-5 \mathrm{a}), 3.82(1 \mathrm{H}, \mathrm{dd}, J 11.8,1.76 \mathrm{~Hz}, \mathrm{H}-5 \mathrm{~b}), 3.71\left(1 \mathrm{H}, \mathrm{m}, \mathrm{OCH}-\left(\mathrm{CH}_{2}\right)_{6} \mathrm{CH}_{3}\right), 3.44(1 \mathrm{H}, \mathrm{m}$, $\left.\mathrm{OCH}-\left(\mathrm{CH}_{2}\right)_{6} \mathrm{CH}_{3}\right), \quad 1.59 \quad\left(2 \mathrm{H}, \quad \mathrm{m}, \quad \mathrm{OCHH}-\mathrm{CH}_{2}\left(\mathrm{CH}_{2}\right)_{5} \mathrm{CH}_{3}\right), \quad 1.27 \quad(10 \mathrm{H}, \quad \mathrm{m}, \quad \mathrm{OCHH}-$ $\left.\mathrm{CH}_{2}\left(\mathrm{CH}_{2}\right)_{5} \mathrm{CH}_{3}\right), 0.88\left(3 \mathrm{H}, \mathrm{t}, J 7.0 \mathrm{~Hz}, \mathrm{OCHH}-\mathrm{CH}_{2}\left(\mathrm{CH}_{2}\right)_{5} \mathrm{CH}_{3}\right)$.

Octyl-5- $\boldsymbol{O}$-p-toluenesulfonyl- $\boldsymbol{\alpha}$-D-arabinofuranoside (5a). Compound 4a $(6.39 \mathrm{~g}, 24.4 \mathrm{mM})$ in anhydrous pyridine $(30 \mathrm{~mL})$ was cooled to $0{ }^{\circ} \mathrm{C}$ and added drop wise a solution of $p$ toluenesulphonyl chloride $(5.11 \mathrm{~g}, 26.8 \mathrm{mM})$. After $24 \mathrm{~h}$ at room temperature, the mixture was poured into ice water $(83 \mathrm{~mL})$, and extracted twice with $\mathrm{CH}_{2} \mathrm{Cl}_{2}$. The combined organic layers were washed successively with $1 \mathrm{~N} \mathrm{HCl}$, aq. $\mathrm{NaHCO}_{3}$, water, dried $\left(\mathrm{MgSO}_{4}\right)$, filtered, and concentrated to give crude yellow oil. Purification by silica gel chromatography (cyclohexane : 
EtOAc, $1: 1)$ gave 5a $(5.72 \mathrm{~g}, 57 \%)$ as a clear oil. FAB-MS $m / z 417(\mathrm{M}+\mathrm{H})^{+} .{ }^{1} \mathrm{H}$ NMR : $\delta 7.78$ $(2 \mathrm{H}, \mathrm{d}, J 8.1 \mathrm{~Hz}, \mathrm{ArH}$, ortho), $7.50(2 \mathrm{H}, \mathrm{d}, J 8.5 \mathrm{~Hz}, \mathrm{ArH}$, meta), $5.42(1 \mathrm{H}, \mathrm{d}, J 5.3 \mathrm{~Hz}, 2-\mathrm{OH})$, $5.32(1 \mathrm{H}, \mathrm{d}, J 5.3 \mathrm{~Hz}, 3-\mathrm{OH}), 4.64(1 \mathrm{H}, \mathrm{d}, J 0.8 \mathrm{~Hz}, \mathrm{H}-1), 4.14$ (1H, dd, J 11.0, $2.6 \mathrm{~Hz}, \mathrm{H}-5 \mathrm{a})$, 4.02 (1H, dd, J 11.0, 6.6 Hz, H-5b), 3.80 (1H, m, H-3), 3.72 (1H, m, H-2), 3.58 (1H, m, H-4), $3.48\left(1 \mathrm{H}, \mathrm{m},-\mathrm{OCHH}\left(\mathrm{CH}_{2}\right)_{6} \mathrm{CH}_{3}\right), 3.40(\mathrm{~s}, 3 \mathrm{H}, \mathrm{ArCH})_{3}, 3.25\left(1 \mathrm{H}, \mathrm{m},-\mathrm{OCH} H\left(\mathrm{CH}_{2}\right)_{6} \mathrm{CH}_{3}\right), 1.49$ $\left(2 \mathrm{H}, \mathrm{m},-\mathrm{OCH}_{2} \mathrm{CH}_{2}\left(\mathrm{CH}_{2}\right) 5 \mathrm{CH} 3\right), 1.26\left(10 \mathrm{H}, \mathrm{m},-\mathrm{OCH}_{2} \mathrm{CH}_{2}\left(\mathrm{CH}_{2}\right)_{5} \mathrm{CH}_{3}\right), 0.85(3 \mathrm{H}, \mathrm{t}, J 7.0 \mathrm{~Hz}$, $\left.\mathrm{OCH}_{2} \mathrm{CH}_{2}\left(\mathrm{CH}_{2}\right)_{5} \mathrm{CH}_{3}\right)$.

Octyl-2,3-di- $\boldsymbol{O}$-acetyl-5- $\boldsymbol{O}$ - $\boldsymbol{p}$-toluenesulfonyl- $\boldsymbol{\alpha}$-D-arabinofuranoside (6a). To an ice cold solution of $5 \mathbf{a}(4.93 \mathrm{~g}, 11.85 \mathrm{mM})$ in pyridine $(21 \mathrm{~mL})$ was added drop wise $\mathrm{Ac}_{2} \mathrm{O}(15.2 \mathrm{~mL})$ and allowed to equilibrate to room temp. Usual work up and purification by silica gel chromatography (cyclohexane : EtOAc, $4: 1$ ) gave $4.0 \mathrm{~g}(68 \%)$ of $6 \mathbf{a}$ as a clear oil. FAB-MS $\mathrm{m} / \mathrm{z}$ $501(\mathrm{M}+\mathrm{H})^{+}$; ${ }^{1} \mathrm{H}$ NMR : $\delta 7.80(2 \mathrm{H}, \mathrm{d}, J 8.3 \mathrm{~Hz}, \mathrm{ArH}$, ortho), 7.35 (2H, d, J 8.5 Hz, ArH, meta), $5.03(1 \mathrm{H}, \mathrm{d}, J 1.3 \mathrm{~Hz}, \mathrm{H}-2), 4.96(1 \mathrm{H}, \mathrm{s}, \mathrm{H}-1), 4.85(1 \mathrm{H}, \mathrm{dd}, J 4.8,1.0 \mathrm{~Hz}, \mathrm{H}-3), 4.27$ (2H, m, H5a,b), $4.15(1 \mathrm{H}, \mathrm{m}, \mathrm{H}-4), 3.62\left(1 \mathrm{H}, \mathrm{m},-\mathrm{OCH}\left(\mathrm{CH}_{2}\right)_{6} \mathrm{CH}_{3}\right), 3.40\left(1 \mathrm{H}, \mathrm{m},-\mathrm{OCH}\left(\mathrm{CH}_{2}\right)_{6} \mathrm{CH}_{3}\right)$, $2.45\left(\mathrm{~s}, 3 \mathrm{H}, \mathrm{ArCH}_{3}\right), 2.07,2.06(3 \mathrm{H}$ each, s, $2 \times \mathrm{OAc}), 1.56\left(2 \mathrm{H}, \mathrm{m},-\mathrm{OCH}_{2} \mathrm{CH}_{2}\left(\mathrm{CH}_{2}\right) 5 \mathrm{CH} 3\right)$, $1.27\left(10 \mathrm{H}, \mathrm{m},-\mathrm{OCH}_{2} \mathrm{CH}_{2}\left(\mathrm{CH}_{2}\right)_{5} \mathrm{CH}_{3}\right), 0.87\left(3 \mathrm{H}, \mathrm{t}, J 7.0 \mathrm{~Hz},-\mathrm{OCH}_{2} \mathrm{CH}_{2}\left(\mathrm{CH}_{2}\right)_{5} \mathrm{CH}_{3}\right)$.

Octyl-2,3-di- $O$-acetyl-5-deoxy-5-S-acetyl-5-thio- $\alpha$-D-arabinofuranoside (7a). To a solution of 6a (1.02 g, $2.04 \mathrm{mM})$ in DMF (4 mL) was added potassium thioacetate $(0.60 \mathrm{~g}, 5.26 \mathrm{mM})$ under an atmosphere of argon. After $26 \mathrm{~h}$ at room temperature, the reaction mixture was diluted with ether $(40 \mathrm{~mL})$. The aqueous phase was extracted with ether and the combined organic layers were washed with water, dried $\left(\mathrm{Na}_{2} \mathrm{SO}_{4}\right)$, filtered, and concentrated to give crude orange oil. Purification by silica gel chromatography (cyclohexane : EtOAc, $4: 1)$ gave $0.719 \mathrm{~g}(88 \%)$ of $7 \mathbf{a}$ as a yellow oil. FAB-MS $m / z 405(\mathrm{M}+\mathrm{H})^{+} ;{ }^{1} \mathrm{HNMR}: \delta 5.03(1 \mathrm{H}, \mathrm{d}, J 1.9 \mathrm{~Hz}, \mathrm{H}-2), 4.96(1 \mathrm{H}$, bs, $\mathrm{H}-1), 4.89(1 \mathrm{H}, \mathrm{dd}, J 5.7,1.9 \mathrm{~Hz}, \mathrm{H}-3), 4.16(1 \mathrm{H}, \mathrm{m}, \mathrm{H}-4), 3.65\left(1 \mathrm{H}, \mathrm{m},-\mathrm{OCHH}\left(\mathrm{CH}_{2}\right)_{6} \mathrm{CH}_{3}\right)$, $3.40\left(2 \mathrm{H}, \mathrm{m}, \mathrm{H}-5 \mathrm{a},-\mathrm{OCH} H\left(\mathrm{CH}_{2}\right)_{6} \mathrm{CH}_{3}\right), 3.17$ (1H, dd, J 15.8, 6.8 Hz, H-5b), 2.36 (s, 3H, -SAc), 2.109, $2.101(3 \mathrm{H}, \mathrm{s}, 2 \times \mathrm{OAc}), 1.56\left(2 \mathrm{H}, \mathrm{m},-\mathrm{OCH}_{2} \mathrm{CH}_{2}\left(\mathrm{CH}_{2}\right)_{5} \mathrm{CH}_{3}\right), 1.27(10 \mathrm{H}, \mathrm{m}$, $\left.\mathrm{OCH}_{2} \mathrm{CH}_{2}\left(\mathrm{CH}_{2}\right)_{5} \mathrm{CH}_{3}\right), 0.88\left(3 \mathrm{H}, \mathrm{t}, J 6.4 \mathrm{~Hz},-\mathrm{OCH}_{2} \mathrm{CH}_{2}\left(\mathrm{CH}_{2}\right)_{5} \mathrm{CH}_{3}\right)$.

\section{Octyl-5-deoxy-5-S-(2,3,5-tri- $O$-benzoyl- $\alpha$-D-arabinofuranosyl)-5-thio- $\alpha$-D-arabinofurano-} side (8a). To a solution of 7a $(1.14 \mathrm{~g}, 2.8 \mathrm{mM})$ in dry $\mathrm{MeOH}(24 \mathrm{~mL})$, cooled to $-40^{\circ} \mathrm{C}$, was added a solution of sodium metal $(65 \mathrm{mg})$ in dry $\mathrm{MeOH}(20 \mathrm{~mL})$. The mixture was stirred for $1 \mathrm{~h}$ at $-40{ }^{\circ} \mathrm{C}$, and then for $30 \mathrm{~min}$ at $0{ }^{\circ} \mathrm{C}$, the course of the reaction being monitored by T.L.C. The solvent was removed by passing argon into the mixture. To the residue was added dry benzene $(34 \mathrm{~mL})$ and nitromethane $(34 \mathrm{~mL})$. The volume was reduced by half under reduced pressure. Then a solution of $2(2.27 \mathrm{~g}, 4.3 \mathrm{mM})$ in benzene $(5 \mathrm{~mL})$ and nitromethane $(5 \mathrm{~mL})$ was added, followed by $\mathrm{Hg}(\mathrm{CN})_{2}(1.08 \mathrm{~g}, 4.3 \mathrm{mM})$ and $4 \mathrm{~A}^{0}$ molecular sieves. The cloudy white reaction mixture was stirred for $40 \mathrm{~h}$ at $65^{\circ} \mathrm{C}$, under an atmosphere of argon, with exclusion of moisture and light. The reaction mixture was allowed to cool, diluted with $\mathrm{CHCl}_{3}$ (24mL), and filtered through a bed of celite. The filtrate was washed with water, aq. $\mathrm{NaHCO}_{3}$, aq. $\mathrm{NaCl}$, dried $\left(\mathrm{MgSO}_{4}\right)$, filtered, and concentrated to give crude yellow foam. Purification by silica gel chromatography (cyclohexane : EtOAc, $3: 1)$ gave $0.28 \mathrm{~g}(15 \%)$ of $\mathbf{8 a}$ as a yellow oil. FAB-MS 
$m / z 729(\mathrm{M}+\mathrm{Li})^{+} ;{ }^{1} \mathrm{HNMR}: \delta 7.70(15 \mathrm{H}, \mathrm{m}, \mathrm{ArH}), 5.81,\left(1 \mathrm{H}, \mathrm{bs}, \mathrm{H}-1^{\prime}\right), 5.65(1 \mathrm{H}, \mathrm{d}, J 4.6 \mathrm{~Hz}, \mathrm{H}-$ 2'), 5.50 (1H, dd, J 1.3, $\left.1.1 \mathrm{~Hz}, \mathrm{H}-3^{\prime}\right), 4.98$ (1H, bs, H-1), 4.77 (3H, m, H-4', H-5'a,b), 4.32 (1H, m, H-4), $4.06\left(1 \mathrm{H}, \mathrm{d}, J_{2, \mathrm{OH}}=9.0 \mathrm{~Hz}, \mathrm{H}-2\right), 3.98\left(1 \mathrm{H}, \mathrm{dd}, J_{3,4, \mathrm{OH}}=10.1,2.9 \mathrm{~Hz}, \mathrm{H}-3\right), 3.65(1 \mathrm{H}, \mathrm{m}$, $\left.\mathrm{OCHH}\left(\mathrm{CH}_{2}\right)_{6} \mathrm{CH}_{3}\right), 3.38\left(1 \mathrm{H}, \mathrm{m},-\mathrm{OCHH}\left(\mathrm{CH}_{2}\right)_{6} \mathrm{CH}_{3}\right), 3.26(1 \mathrm{H}, \mathrm{dd}, J$ 14.7, $4.8 \mathrm{~Hz}, \mathrm{H}-5 \mathrm{a}), 3.01$ $(2 \mathrm{H}, \mathrm{m}, 2-\mathrm{OH}, \mathrm{H}-5 \mathrm{~b}), 2.60(1 \mathrm{H}, \mathrm{d}, J 10.1 \mathrm{~Hz}, 3-\mathrm{OH}), 1.52\left(2 \mathrm{H}, \mathrm{m},-\mathrm{OCH}_{2} \mathrm{CH}_{2}\left(\mathrm{CH}_{2}\right)_{5} \mathrm{CH}_{3}\right), 1.25$ $\left(10 \mathrm{H}, \mathrm{m},-\mathrm{OCH}_{2} \mathrm{CH}_{2}\left(\mathrm{CH}_{2}\right)_{5} \mathrm{CH}_{3}\right), 0.86\left(3 \mathrm{H}, \mathrm{t}, J 6.4 \mathrm{~Hz},-\mathrm{OCH}_{2} \mathrm{CH}_{2}\left(\mathrm{CH}_{2}\right)_{5} \mathrm{CH}_{3}\right)$. (HRMS calcd for $\mathrm{C}_{39} \mathrm{H}_{46} \mathrm{O}_{11} \mathrm{~S}: m / z(\mathrm{M}+\mathrm{Na})^{+}$745.2653. Found $m / z$ 745.2678).

Octyl-5-deoxy-5-S-( $\alpha$-D-arabinofuranosyl)-5-thio- $\alpha$-D-arabinofuranoside (9a). To a solution of $8 \mathbf{a}(0.18 \mathrm{~g}, 0.25 \mathrm{mM})$ in dry chloroform $(2 \mathrm{~mL})$ and dry methanol $(1.5 \mathrm{~mL})$ was added a solution of sodium metal $(10 \mathrm{mg})$ in dry methanol $(1 \mathrm{~mL})$. The reaction mixture was stirred under an atmosphere of argon. After $36 \mathrm{~h}$ at room temperature, the reaction mixture was diluted with water $(6 \mathrm{~mL})$ and neutralized with Dowex-50W (50X8-400) cation exchange resin. The mixture was filtered through a cintered glass funnel and the filtrate partitioned between chloroform and water. The aqueous layer was washed with chloroform and then concentrated under reduced pressure. The white residue was purified by silica gel chromatography $\left(\mathrm{CHCl}_{3}\right.$ : $\mathrm{MeOH}, 9: 1)$ to give $0.04 \mathrm{~g}(40 \%)$ of 9a as a clear oil. FAB-MS m/z $417(\mathrm{M}+\mathrm{Li})^{+}$; ${ }^{1} \mathrm{HNMR}$ $\left(\mathrm{Me}_{2} \mathrm{SO}-d_{6}\right): \delta 5.53(1 \mathrm{H}, \mathrm{d}, J 5.5 \mathrm{~Hz}, \mathrm{OH}), 5.37(1 \mathrm{H}, \mathrm{d}, J 4.8 \mathrm{~Hz}, \mathrm{OH}), 5.23(1 \mathrm{H}, \mathrm{d}, J 5.1 \mathrm{~Hz}$, $\mathrm{OH}), 5.19(1 \mathrm{H}, \mathrm{d}, J 5.5 \mathrm{~Hz}, \mathrm{OH}), 4.96(1 \mathrm{H}, \mathrm{d}, J 3.7 \mathrm{~Hz}, \mathrm{H}-1), 4.75\left(1 \mathrm{H}, \mathrm{t}, J 5.5 \mathrm{~Hz}, 5^{\prime}-\mathrm{OH}\right), 4.68$ (1H, d, J 2.0 Hz, H-1') 3.85 (1H, m, H-3), 3.65 (8H, m, H-5'a, H-4', H-3', H-2', H-2, H-3, $\left.\mathrm{OCH}\left(\mathrm{CH}_{2}\right)_{6} \mathrm{CH}_{3}\right), 3.44\left(1 \mathrm{H}, \mathrm{m}, \mathrm{H}-5^{\prime} \mathrm{b}\right), 2.91(1 \mathrm{H}, \mathrm{dd}, J 13.8,3.7 \mathrm{~Hz}, \mathrm{H}-5 \mathrm{a}), 2.65$ (1H, dd, $J$ 13.8, 7.0 Hz, H-5b), $1.50\left(2 \mathrm{H}, \mathrm{m},-\mathrm{OCH}_{2} \mathrm{CH}_{2}\left(\mathrm{CH}_{2}\right)_{5} \mathrm{CH}_{3}\right), 1.25\left(10 \mathrm{H}, \mathrm{m},-\mathrm{OCH}_{2} \mathrm{CH}_{2}\left(\mathrm{CH}_{2}\right)_{5} \mathrm{CH}_{3}\right)$, $0.85\left(3 \mathrm{H}, \mathrm{t}, J 6.4 \mathrm{~Hz},-\mathrm{OCH}_{2} \mathrm{CH}_{2}\left(\mathrm{CH}_{2}\right)_{5} \mathrm{CH}_{3}\right)$. (HRMS calcd for $\mathrm{C}_{18} \mathrm{H}_{34} \mathrm{O}_{8} \mathrm{~S}: \mathrm{m} / z(\mathrm{M}+\mathrm{Na})^{+}$ 433.1866. Found $m / z, 433.1868)$.

1-Methylpropyl-2,3,5-tri- $\boldsymbol{O}$-benzoyl- $\boldsymbol{\alpha}$-D-arabinofuranoside $(\mathbf{3 b})$. To a solution of $\mathbf{2}^{16}(2.71 \mathrm{~g}$, $5.17 \mathrm{mM})$ in $\mathrm{CH}_{3} \mathrm{CN}(80 \mathrm{~mL})$ was added n-octanol $(0.81 \mathrm{~mL}, 8.9 \mathrm{mM})$ and $\mathrm{SnCl}_{4}(1.38 \mathrm{~mL}, 11.8$ $\mathrm{mM}$ ) as done in 3a to give an orange oil. Purification by silica gel chromatography (cyclohexane : EtOAc, $4: 1)$ gave of $\mathbf{3 b}$ as diastereomers $(2.0 \mathrm{~g}, 75 \%)$ as a clear oil. FAB-MS $m / z 525$ $(\mathrm{M}+\mathrm{Li})^{+} ;{ }^{1} \mathrm{H}$ NMR : $\delta 8.70(15 \mathrm{H}, \mathrm{m}, \mathrm{ArH}), 5.58(1 \mathrm{H}, \mathrm{bs}, \mathrm{H}-3), 5.48(1 \mathrm{H}, \mathrm{bs}, \mathrm{H}-2), 5.40(1 \mathrm{H}, \mathrm{bs}$, $\mathrm{H}-1), 4.87$ (1H, m, H-5a), 4.67 (1H, m, H-5b), $4.57(1 \mathrm{H}, \mathrm{m}, \mathrm{H}-4), 3.81(1 \mathrm{H}, \mathrm{m}$, $\left.\mathrm{OCH}\left(\mathrm{CH}_{3}\right) \mathrm{CH}_{2} \mathrm{CH}_{3}\right), 1.58\left(2 \mathrm{H}, \mathrm{m}, \mathrm{OCH}\left(\mathrm{CH}_{3}\right) \mathrm{CH}_{2} \mathrm{CH}_{3}\right), 1.26\left(3 \mathrm{H}, \mathrm{d}, \mathrm{OCH}\left(\mathrm{CH}_{3}\right) \mathrm{CH}_{2} \mathrm{CH}_{3}\right), 0.88$ $\left(3 \mathrm{H}, \mathrm{t}, J 6.8 \mathrm{~Hz}, \mathrm{OCH}\left(\mathrm{CH}_{3}\right) \mathrm{CH}_{2} \mathrm{CH}_{3}\right)$.

1-Methylpropyl- $\alpha$-D-arabinofuranoside (4b). A solution of $\mathbf{3 b}(3.29 \mathrm{~g}, 6.35 \mathrm{mM})$ in dry methanol $(28 \mathrm{~mL})$ was treated with methanolic ammonia $(7 \mathrm{~N}, 28 \mathrm{~mL})$ under an atmosphere of argon as reported earlier in $\mathbf{4 a}$ to give $\mathbf{4 b}(1.3 \mathrm{~g})$ as a yellow oil. The material was used directly in the next step.

1-Methylpropyl-5- $O$-p-toluenesulfonyl- $\alpha$-D-arabinofuranoside $(5 \mathrm{~b})$. Compound $4 \mathrm{~b}$ (1.3 g, $6.31 \mathrm{mM})$ in anhydrous pyridine $(9.3 \mathrm{~mL})$ was cooled to $0{ }^{\circ} \mathrm{C}$ and added drop wise a solution of p-toluenesulphonyl chloride $(1.30 \mathrm{~g}, 6.82 \mathrm{mM})$ as mentioned earlier in 5a to give a crude yellow oil. Purification by silica gel chromatography (cyclohexane : EtOAc, $1: 1$ ) gave $\mathbf{5 b}$ as diastereomers $(1.4 \mathrm{~g}, 62 \%)$ as a clear oil. FAB-MS $m / z, 361(\mathrm{M}+\mathrm{H})^{+} .{ }^{1} \mathrm{HNMR}: \delta 7.80(2 \mathrm{H}, \mathrm{d}, J$ 
$8.3 \mathrm{~Hz}, \mathrm{ArH}$, ortho), 7.35 (2H, d, J 8.3 Hz, ArH, meta), 5.08 (1H, s, H-1), 4.22 (2H, m, H-5a,b), $4.05(1 \mathrm{H}, \mathrm{m}, \mathrm{H}-2), 3.88(1 \mathrm{H}, \mathrm{m}, \mathrm{H}-3), 3.70\left(1 \mathrm{H}, \mathrm{m},-\mathrm{OCH}\left(\mathrm{CH}_{3}\right) \mathrm{CH}_{2} \mathrm{CH}_{3}\right), 2.87(1 \mathrm{H}, J 10.7 \mathrm{~Hz}$, 3-OH), $2.45\left(3 \mathrm{H}, \mathrm{s}, \mathrm{ArCH}_{3}\right), 2.22(1 \mathrm{H}, J 7.7 \mathrm{~Hz}, 2-\mathrm{OH}), 1.46\left(2 \mathrm{H}, \mathrm{m},-\mathrm{OCH}\left(\mathrm{CH}_{3}\right) \mathrm{CH}_{2} \mathrm{CH}_{3}\right), 1.12$ $\left(3 \mathrm{H}, \mathrm{m}, \mathrm{OCH}\left(\mathrm{CH}_{3}\right) \mathrm{CH}_{2} \mathrm{CH}_{3}\right), 0.87\left(3 \mathrm{H}, \mathrm{t}, J 7.2 \mathrm{~Hz}, \mathrm{OCH}\left(\mathrm{CH}_{3}\right) \mathrm{CH}_{2} \mathrm{CH}_{3}\right)$.

1-Methylpropyl-2,3-di- $O$-acetyl-5- $O$-p-toluenesulfonyl- $\alpha$-D-arabinofuranoside (6b). To an ice cold solution of $\mathbf{5 b}(1.62 \mathrm{~g}, 4.50 \mathrm{mM})$ in pyridine $(8 \mathrm{~mL})$ was added drop wise $\mathrm{Ac}_{2} \mathrm{O}(7.3$ $\mathrm{mL})$ as done in 6a to give a crude oil. Purification by silica gel chromatography (cyclohexane/EtOAc, $3: 1)$ gave $1.48 \mathrm{~g}$ (74\%) of $\mathbf{6 b}$ as diastereomers. FAB-MS m/z 451 $(\mathrm{M}+\mathrm{Li})^{+} ;{ }^{1} \mathrm{H}$ NMR : $\delta 7.80(2 \mathrm{H}, \mathrm{ArH}$, ortho), $7.34(2 \mathrm{H}, \mathrm{ArH}$, meta), $5.07(1 \mathrm{H}, \mathrm{s}, \mathrm{H}-1), 5.00(1 \mathrm{H}$, bs, H-3), 4.88 (1H, m, H-2), $4.26(3 \mathrm{H}, \mathrm{m}, \mathrm{H}-4, \mathrm{H}-5 \mathrm{a}, \mathrm{b}), 3.65\left(1 \mathrm{H}, \mathrm{m},-\mathrm{OCH}\left(\mathrm{CH}_{3}\right) \mathrm{CH}_{2} \mathrm{CH}_{3}\right), 2.45$ $\left(3 \mathrm{H}, \mathrm{s}, \mathrm{ArCH}_{3}\right), 2.07(6 \mathrm{H}, \mathrm{s}, 2 \times \mathrm{OAc}), 1.47\left(2 \mathrm{H}, \mathrm{m},-\mathrm{OCH}\left(\mathrm{CH}_{3}\right) \mathrm{CH}_{2} \mathrm{CH}_{3}\right), 1.13(3 \mathrm{H}, \mathrm{m}$, $\left.\mathrm{OCH}\left(\mathrm{CH}_{3}\right) \mathrm{CH}_{2} \mathrm{CH}_{3}\right), 0.90\left(3 \mathrm{H}, \mathrm{t}, J 7.2 \mathrm{~Hz}, \mathrm{OCH}\left(\mathrm{CH}_{3}\right) \mathrm{CH}_{2} \mathrm{CH}_{3}\right)$. Anal. Calcd. for $\mathrm{C}_{20} \mathrm{H}_{28} \mathrm{O}_{9} \mathrm{~S}$. $0.1 \mathrm{CHCl}_{3}: \mathrm{C}, 52.89 ; \mathrm{H}, 6.20$. Found : $\mathrm{C}, 52.68 ; \mathrm{H}, 6.19$.

1-Methylpropyl-2,3-di-O-acetyl-5-deoxy-5-S-acetyl-5-thio- $\alpha$-D-arabinofuranoside (7b). To a solution of $\mathbf{6 b}(1.09 \mathrm{~g}, 2.18 \mathrm{mM})$ in DMF $(4.3 \mathrm{~mL})$ was added potassium thioacetate $(0.64 \mathrm{~g}$, $5.62 \mathrm{mM}$ ) as reported in $7 \mathbf{a}$ to give a crude orange oil. Purification by silica gel chromatography (cyclohexane : EtOAc, $4: 1)$ gave $0.689 \mathrm{~g}(81 \%)$ of $\mathbf{7 b}$ as a yellow oil (diastereomers). FAB-MS m/z $355(\mathrm{M}+\mathrm{Li})^{+}$; ${ }^{1} \mathrm{HNMR}: \delta 5.09(1 \mathrm{H}, \mathrm{s}, \mathrm{H}-1), 5.00(1 \mathrm{H}, \mathrm{s}, \mathrm{H}-3), 4.86(1 \mathrm{H}, \mathrm{m}, \mathrm{H}-2), 4.23$ (3H, m, H-4, H-5a,b), $3.63\left(1 \mathrm{H}, \mathrm{m},-\mathrm{CH}\left(\mathrm{CH}_{3}\right) \mathrm{CH}_{2} \mathrm{CH}_{3}\right), 2.45\left(3 \mathrm{H}, \mathrm{s}, \mathrm{ArCH}_{3}\right), 2.07$ (6H, s, $\left.2 \times \mathrm{OAc}\right)$, $1.47\left(2 \mathrm{H}, \mathrm{m},-\mathrm{CH}\left(\mathrm{CH}_{3}\right) \mathrm{CH}_{2} \mathrm{CH}_{3}\right), 1.22\left(6 \mathrm{H}, \mathrm{m},-\mathrm{CH}\left(\mathrm{CH}_{3}\right) \mathrm{CH}_{2} \mathrm{CH}_{3}\right)$. Anal. Calcd. for $\mathrm{C}_{15} \mathrm{H}_{24} \mathrm{O}_{7} \mathrm{~S}$ : C, 51.71; H, 6.94. Found : C, 51.37; H, 6.85.

1-Methylpropyl-5-deoxy-5-S-(2,3,5-tri- $O$-benzoyl- $\alpha$-D-arabinofuranosyl)-5-thio- $\alpha$-D-arabinofuranoside $(\mathbf{8 b})$. To a solution of $7 \mathbf{b}(0.868 \mathrm{~g}, 2.49 \mathrm{mM})$ in dry $\mathrm{MeOH}(18 \mathrm{~mL})$, cooled to -40 ${ }^{\circ} \mathrm{C}$, was added a solution of sodium metal $(60 \mathrm{mg})$ in dry $\mathrm{MeOH}(18 \mathrm{~mL})$. The mixture was stirred at $-40^{\circ} \mathrm{C}$, and slowly allowed to equilibrate to room temperature. After $20 \mathrm{~h}$ the solvent was removed by passing argon into the mixture, and then carefully under reduced pressure. To the residue was added dry benzene $(30 \mathrm{~mL})$ and nitromethane $(30 \mathrm{~mL})$. The volume was reduced by half under reduced pressure. Then a solution of $3(2.09 \mathrm{~g}, .3 .97 \mathrm{mM})$ in benzene / nitromethane $\left(4.5 \mathrm{~mL}\right.$ each) was added, followed by $\mathrm{Hg}(\mathrm{CN})_{2}(1.0 \mathrm{~g}, 3.97 \mathrm{mM})$ and $4 \mathrm{~A}^{0}$ molecular sieves as mentioned in 8a to give a crude yellow oil. Purification by silica gel chromatography (cyclohexane : EtOAc, $3: 1$ to $100 \%$ EtOAc) gave $0.70 \mathrm{~g}(43 \%)$ of $\mathbf{8 b}$ as a pale yellow oil (diastereomers). FAB-MS $m / z 673(\mathrm{M}+\mathrm{Li})^{+} ;{ }^{1} \mathrm{HNMR}: \delta 7.70(15 \mathrm{H}, \mathrm{m}, \mathrm{ArH}), 5.82$ $\left(1 \mathrm{H}, \mathrm{bs}, \mathrm{H}-1^{\prime}\right), 5.65\left(1 \mathrm{H}, \mathrm{d}, J 4.6 \mathrm{~Hz}, \mathrm{H}-3^{\prime}\right), 5.50\left(1 \mathrm{H}, \mathrm{dd}, J 1.3,1.1 \mathrm{~Hz}, \mathrm{H}-2^{\prime}\right), 5.11(1 \mathrm{H}, \mathrm{bs}, \mathrm{H}-$ 1), 4.77 (3H, m, H-4', H-5'a,b), $4.32(1 \mathrm{H}, \mathrm{m}, \mathrm{H}-4), 4.03\left(1 \mathrm{H}, \mathrm{d}, J_{2,3, O H}=9.2,3.3 \mathrm{~Hz}, \mathrm{H}-2\right), 3.98$ $(1 \mathrm{H}, \mathrm{m}, \mathrm{H}-3), 3.65\left(1 \mathrm{H}, \mathrm{m},-\mathrm{CH}\left(\mathrm{CH}_{3}\right) \mathrm{CH}_{2} \mathrm{CH}_{3}\right), 3.25(1 \mathrm{H}, \mathrm{dd}, J 14.5,5.0 \mathrm{~Hz}, \mathrm{H}-5 \mathrm{a}), 3.01(2 \mathrm{H}$, m, 2-OH, H-5b), $2.63(1 \mathrm{H}, \mathrm{d}, J 10.7 \mathrm{~Hz}, 3-\mathrm{OH}), 1.52\left(2 \mathrm{H}, \mathrm{m},-\mathrm{OCH}\left(\mathrm{CH}_{3}\right) \mathrm{CH}_{2} \mathrm{CH}_{3}\right), 1.25(3 \mathrm{H}, \mathrm{m}$, $\left.-\mathrm{CH}\left(\mathrm{CH}_{3}\right) \mathrm{CH}_{2} \mathrm{CH}_{3}\right), 0.86\left(3 \mathrm{H}, \mathrm{t}, 6.8 \mathrm{~Hz},-\mathrm{CH}\left(\mathrm{CH}_{3}\right) \mathrm{CH}_{2} \mathrm{CH}_{3}\right)$. (HRMS calcd for $\mathrm{C}_{35} \mathrm{H}_{38} \mathrm{O}_{11} \mathrm{~S}: \mathrm{m} / z$ $(\mathrm{M}+\mathrm{Na})^{+}$689.2027. Found $m / z$ 689.2033).

1-Methylpropyl-5-deoxy-5- $S$-( $\alpha$-D-arabinofuranosyl)-5-thio- $\alpha$-D-arabinofuranoside (9b). To a solution of $\mathbf{8 b}(0.649 \mathrm{~g}, 0.97 \mathrm{mM})$ in dry chloroform $(7 \mathrm{~mL})$ and dry $\mathrm{MeOH}(7 \mathrm{~mL})$ was 
added a solution of sodium metal $(60 \mathrm{mg})$ in dry $\mathrm{MeOH}(4 \mathrm{~mL})$ as in 9 a followed by gel chromatography $\left(\mathrm{CHCl}_{3}: \mathrm{MeOH}, 9: 1\right)$ to give $0.209 \mathrm{~g}(61 \%)$ of $\mathbf{9 b}$ as a clear oil (diastereomers). FAB-MS $m / z 361(\mathrm{M}+\mathrm{Li})^{+} ;{ }^{1} \mathrm{HNMR}\left(\mathrm{Me}_{2} \mathrm{SO}-d_{6}\right): \delta 5.53(1 \mathrm{H}, \mathrm{d}, J 5.5 \mathrm{~Hz}, \mathrm{OH})$, $5.37(1 \mathrm{H}, \mathrm{d}, 5.0 \mathrm{~Hz}, \mathrm{OH}), 5.23(1 \mathrm{H}, \mathrm{d}, J 5.0 \mathrm{~Hz}, \mathrm{OH}), 5.18(1 \mathrm{H}, \mathrm{dd}, J 5.7 \mathrm{~Hz}, \mathrm{OH}), 4.96(1 \mathrm{H}, \mathrm{d}, J$ $1.7 \mathrm{~Hz}, \mathrm{H}-1), 4.75$ (1H, t, J 5.2 Hz, 5'-OH), 4.68 (1H, d, J 1.9 Hz, H-1'), 3.85 (1H, m, H-3), 3.65 (7H, m, H-5'a, H-4', H-3', H-2', H-2, H-3, -CH(CH3) $\left.\mathrm{CH}_{2} \mathrm{CH}_{3}\right), 3.44$ (1H, m, H-5'b), 2.92 (1H, dd, J 13.4, $3.7 \mathrm{~Hz}, \mathrm{H}-5 \mathrm{a}), 2.65$ (1H, dd, J 13.4, 7.2 Hz, H-5b), 1.50 (2H, m, $\left.\mathrm{OCH}\left(\mathrm{CH}_{3}\right) \mathrm{CH}_{2} \mathrm{CH}_{3}\right), \quad 1.25\left(3 \mathrm{H}, \quad \mathrm{m}, \quad-\mathrm{CH}\left(\mathrm{CH}_{3}\right) \mathrm{CH}_{2} \mathrm{CH}_{3}\right), \quad 0.85 \quad(3 \mathrm{H}, \quad \mathrm{t}, \quad J \quad 7.2 \mathrm{~Hz}, \quad-$ $\left.\mathrm{CH}\left(\mathrm{CH}_{3}\right) \mathrm{CH}_{2} \mathrm{CH}_{3}\right)$. (HRMS calcd for $\mathrm{C}_{14} \mathrm{H}_{26} \mathrm{O}_{8} \mathrm{~S}: \mathrm{m} / z$ ( $\left.\mathrm{M}+\mathrm{Na}\right)^{+} 377.1240$. Found $\mathrm{m} / z$ 377.1244). $\mathbf{3}^{\prime}$-Phenylpropyl-2,3,5-tri- $\boldsymbol{O}$-benzoylarabinofuranoside (3c). To a solution of $\mathbf{2}^{16}$ (9.7 g, 18.5 $\mathrm{mM})$ in $\mathrm{CH}_{3} \mathrm{CN}(289 \mathrm{~mL})$, was added 3-phenyl-1-propanol $(4.3 \mathrm{~mL}, 31.5 \mathrm{mM})$ and $\mathrm{SnCl}_{4}(4.8$ $\mathrm{mL}, 40.8 \mathrm{mM}$ ) as done in 3a to give a crude yellow oil. Purification by silica gel chromatography (cyclohexane : EtOAc, $4: 1)$ gave $6.72 \mathrm{~g}(63 \%)$ of $3 \mathrm{c}$ as a clear oil. FAB-MS $\mathrm{m} / \mathrm{z} 587(\mathrm{M}+\mathrm{Li})^{+}$; ${ }^{1} \mathrm{HNMR}: \delta 7.62(20 \mathrm{H}, \mathrm{m}, \mathrm{Ar} . H), 5.58(1 \mathrm{H}, \mathrm{d}, J 4.8 \mathrm{~Hz}, \mathrm{H}-3), 5.54$ (1H, d, J $\left.1.3 \mathrm{~Hz}, \mathrm{H}-2\right), 5.28$ $(1 \mathrm{H}, \mathrm{s}, \mathrm{H}-1), 4.82$ (1H, dd, J 11.6, $3.5 \mathrm{~Hz}, \mathrm{H}-5 \mathrm{a}), 4.67$ (1H, dd, $J$ 12.1, 5.1 Hz, H-5b), 4.58 (1H, m, H-4), $3.82\left(1 \mathrm{H}, \mathrm{m},-\mathrm{OCH} \mathrm{HCH}_{2} \mathrm{CH}_{2} \mathrm{C}_{6} \mathrm{H}_{5}\right), 3.57\left(1 \mathrm{H}, \mathrm{m},-\mathrm{OCH} \mathrm{HCH}_{2} \mathrm{CH}_{2} \mathrm{C}_{6} \mathrm{H}_{5}\right), 2.72(2 \mathrm{H}, \mathrm{m},-$ $\left.\mathrm{OCH}_{2} \mathrm{CH}_{2} \mathrm{CH}_{2} \mathrm{C}_{6} \mathrm{H}_{5}\right), 2.00\left(2 \mathrm{H}, \mathrm{m},-\mathrm{OCH}_{2} \mathrm{CH}_{2} \mathrm{CH}_{2} \mathrm{C}_{6} \mathrm{H}_{5}\right)$. Anal. Calcd. for $\mathrm{C}_{35} \mathrm{H}_{32} \mathrm{O}_{8} \mathrm{H}_{2} \mathrm{O}$ : C, 70.23; H, 5.68. Found : C, 70.09; H, 5.58.

3'-Phenylpropyl- $\alpha$-D-arabinofuranoside (4c). A solution of $3 \mathbf{c}(6.7 \mathrm{~g}, 11.5 \mathrm{mM})$ in $51 \mathrm{~mL}$ of dry $\mathrm{MeOH}$ was treated with methanolic ammonia $(7 \mathrm{~N}, 51 \mathrm{~mL})$ as reported in $4 \mathbf{a}$ to give $3.0 \mathrm{~g}$ (quant.) of $\mathbf{4 c}$ as a yellow oil. The material was used directly in the next step.

3'-Phenylpropyl-5-O $-\boldsymbol{p}$-toluenesulfonyl- $\boldsymbol{\alpha}$-D-arabinofuranoside $(\mathbf{5 c})$. Compound $\mathbf{4 c}(3.0 \mathrm{~g}$, $11.5 \mathrm{mM})$ in anhydrous pyridine $(14 \mathrm{~mL})$ was cooled to $0{ }^{\circ} \mathrm{C}$ and added drop wise a solution of p-toluenesulphonyl chloride $(2.42 \mathrm{~g}, 12.7 \mathrm{mM})$ as done in $\mathbf{5 a}$ to give an orange-brown oil. Purification by silica gel chromatography (cyclohexane : EtOAc, $1: 1)$ gave $2.91 \mathrm{~g}(60 \%)$ of 5c as a pale yellow oil. FAB-MS $m / z, 423(\mathrm{M}+\mathrm{H})^{+} ;{ }^{1} \mathrm{HNMR}: \delta 7.80(2 \mathrm{H}, \mathrm{d}, J 8.6 \mathrm{~Hz}, \mathrm{ArH}), 7.32$ $(2 \mathrm{H}, \mathrm{d}, J 8.6 \mathrm{~Hz}, \mathrm{ArH}), 7.25$ (5H, m, - $\left.\mathrm{OCHHCH}_{2} \mathrm{CH}_{2} \mathrm{C}_{6} \mathrm{H}_{5}\right), 4.94(1 \mathrm{H}, \mathrm{s}, \mathrm{H}-1), 4.27$ (3H, m, H-4, $\mathrm{H}-5 \mathrm{a}, \mathrm{b}), \quad 3.92(1 \mathrm{H}, \quad \mathrm{m}, \mathrm{H}-3), 3.73\left(1 \mathrm{H}, \quad \mathrm{m},-\mathrm{OCH}_{\mathrm{HCH}} \mathrm{CH}_{2} \mathrm{C}_{6} \mathrm{H}_{5}\right), 3.43(1 \mathrm{H}, \mathrm{m}, \quad-$ $\mathrm{OCH}_{\left.\mathrm{CCH}_{2} \mathrm{CH}_{2} \mathrm{C}_{6} \mathrm{H}_{5}\right),} 2.74(1 \mathrm{H}, \quad \mathrm{d}, \quad J \quad 10.1 \mathrm{~Hz}, \quad 3-\mathrm{OH}), 2.65 \quad(2 \mathrm{H}, \quad \mathrm{t}, \quad J \quad 7.9 \mathrm{~Hz}, \quad-$ $\left.\mathrm{OCHHCH}_{2} \mathrm{CH}_{2} \mathrm{C}_{6} \mathrm{H}_{5}\right), 2.44\left(3 \mathrm{H}, \mathrm{s}, \mathrm{ArCH}_{3}\right), 2.36(1 \mathrm{H}, \mathrm{d}, J 6.8 \mathrm{~Hz}, 2-\mathrm{OH}), 1.90$ (2H, m, $\mathrm{OCHHCH}_{2} \mathrm{CH}_{2} \mathrm{C}_{6} \mathrm{H}_{5}$ ). Anal. Calcd. for $\mathrm{C}_{21} \mathrm{H}_{26} \mathrm{O}_{7} \mathrm{~S} .0 .1 \mathrm{CHCl}_{3}$ : C, 60.82; H, 6.22. Found : C, $60.52 ; \mathrm{H}, 6.11$.

3'-Phenylpropyl-2,3-di- $\boldsymbol{O}$-acetyl-5- $\boldsymbol{O}$-p-toluenesulfonyl- $\boldsymbol{\alpha}$-D-arabinofuranoside $(\mathbf{6 c})$. To an ice cold solution of $\mathbf{5 c}(2.78 \mathrm{~g}, 6.59 \mathrm{mM})$ in pyridine $(11.7 \mathrm{~mL})$ was added drop wise $\mathrm{Ac}_{2} \mathrm{O}(10.7$ $\mathrm{mL}$ ) as in 6a to give a crude oil. Purification by silica gel chromatography (cyclohexane : EtOAc, $4: 1)$ gave $1.46 \mathrm{~g}(45 \%)$ of $6 \mathrm{c}$ as a clear oil. FAB-MS $m / z, 507(\mathrm{M}+\mathrm{H})^{+} ;{ }^{1} \mathrm{HNMR}: \delta 7.80$ $(2 \mathrm{H}, \mathrm{d}, J 8.3 \mathrm{~Hz}, \mathrm{ArH}), 7.33(2 \mathrm{H}, \mathrm{d}, J 7.9 \mathrm{~Hz}, \mathrm{ArH}), 7.22\left(5 \mathrm{H}, \mathrm{m},-\mathrm{OCHHCH}_{2} \mathrm{CH}_{2} \mathrm{C}_{6} H_{5}\right), 5.05$ $(1 \mathrm{H}, \mathrm{d}, J 1.3 \mathrm{~Hz}, \mathrm{H}-2), 4.97$ (1H, s, H-1), 4.88 (1H, dd, J 4.8, $0.9 \mathrm{~Hz}, \mathrm{H}-3), 4.26$ (2H, m, H-5a,b), $4.19(1 \mathrm{H}, \mathrm{m}, \mathrm{H}-4), 3.65\left(1 \mathrm{H}, \mathrm{m},-\mathrm{OCH} \mathrm{HCH}_{2} \mathrm{CH}_{2} \mathrm{C}_{6} \mathrm{H}_{5}\right), 3.40\left(1 \mathrm{H}, \mathrm{m},-\mathrm{OCH}^{2} \mathrm{CH}_{2} \mathrm{CH}_{2} \mathrm{C}_{6} H_{5}\right)$, 
$2.65\left(2 \mathrm{H}, \mathrm{m},-\mathrm{OCHHCH}_{2} \mathrm{CH}_{2} \mathrm{C}_{6} \mathrm{H}_{5}\right), 2.44\left(3 \mathrm{H}, \mathrm{s}, \mathrm{Ar} . \mathrm{CH}_{3}\right), 2.08(6 \mathrm{H}, \mathrm{s}, 2 \times \mathrm{OAc}), 1.88(2 \mathrm{H}, \mathrm{m},-$ $\mathrm{OCHHCH}_{2} \mathrm{CH}_{2} \mathrm{C}_{6} \mathrm{H}_{5}$ ); Anal. Calcd. for $\mathrm{C}_{25} \mathrm{H}_{30} \mathrm{O}_{9} \mathrm{~S}: \mathrm{C}, 59.29 ; \mathrm{H}, 5.93$. Found : C, 59.45; H, 6.30. 3'-Phenylpropyl-2,3-di- $O$-acetyl-5-deoxy-5-S-acetyl- $\alpha$-D-arabinofuranoside $(7 \mathrm{c})$. To a solution of 6c $(1.41 \mathrm{~g}, 2.80 \mathrm{mM})$ in DMF $(6.5 \mathrm{~mL})$ was added potassium thioacetate $(1.07 \mathrm{~g}, 9.38 \mathrm{mM})$ as reported in $7 \mathbf{a}$ to give a crude orange oil. Purification by silica gel chromatography (cyclohexane : EtOAc, $4: 1)$ gave $0.904 \mathrm{~g}(79 \%)$ of $7 \mathrm{c}$ as an orange oil. FAB-MS $m / z, 417(\mathrm{M}+\mathrm{Li})^{+} ;{ }^{1} \mathrm{HNMR}$ : $\delta 7.22(5 \mathrm{H}, \mathrm{m}$, Ar.H), $5.06(1 \mathrm{H}, \mathrm{d}, J 1.5 \mathrm{~Hz}, \mathrm{H}-2), 4.96(1 \mathrm{H}, \mathrm{s}, \mathrm{H}-1), 4.90(1 \mathrm{H}, \mathrm{dd}, J 5.5,1.5 \mathrm{~Hz}$, $\mathrm{H}-3), \quad 4.19(1 \mathrm{H}, \quad \mathrm{m}, \mathrm{H}-4), \quad 3.69\left(1 \mathrm{H}, \quad \mathrm{m}, \quad-\mathrm{OCHHCH}_{2} \mathrm{CH}_{2} \mathrm{C}_{6} H_{5}\right), 3.43 \quad(1 \mathrm{H}, \mathrm{m}, \quad-$ OCH $\left.\mathrm{HCH}_{2} \mathrm{CH}_{2} \mathrm{C}_{6} H_{5}\right), 3.36(1 \mathrm{H}$, dd, $J 13.8,4.8 \mathrm{~Hz}, \mathrm{H}-5 \mathrm{a}), 3.16(1 \mathrm{H}, \mathrm{dd}, J 13.8,6.8 \mathrm{~Hz}, \mathrm{H}-5 \mathrm{~b})$, $2.69\left(2 \mathrm{H}, \mathrm{m},-\mathrm{OCHHCH}_{2} \mathrm{CH}_{2} \mathrm{C}_{6} H_{5}\right), 2.35(3 \mathrm{H}, \mathrm{s},-\mathrm{SAc}), 2.11(6 \mathrm{H}, \mathrm{s}, 2 \mathrm{xOAc}), 1.90(2 \mathrm{H}, \mathrm{m},-$ $\mathrm{OCHHCH}_{2} \mathrm{CH}_{2} \mathrm{C}_{6} \mathrm{H}_{5}$ ). Anal. Calcd. for $\mathrm{C}_{20} \mathrm{H}_{26} \mathrm{O}_{7} \mathrm{~S}: \mathrm{C}, 58.52 ; \mathrm{H}, 6.38$. Found : C, 58.79; H, 6.39. 3'-Phenylpropyl-5-deoxy-5- $S$-(2,3,5-tri- $O$-benzoyl- $\alpha$-D-arabinofuranosyl)-5-thio- $\alpha$-D-arabinofuranoside $(\mathbf{8 c})$. To a solution of $7 \mathrm{c}(0.848 \mathrm{~g}, 2.06 \mathrm{mM})$ in dry $\mathrm{MeOH}(15 \mathrm{~mL})$, cooled to $40{ }^{\circ} \mathrm{C}$, was added a solution of sodium metal $(70 \mathrm{mg})$ in dry $\mathrm{MeOH}(15 \mathrm{~mL})$. The mixture was stirred at $-40^{\circ} \mathrm{C}$, and slowly allowed to equilibrate to room temperature. After $18 \mathrm{~h}$ the solvent was removed by passing argon into the mixture, and then carefully under reduced pressure. To the residue was added dry benzene $(25 \mathrm{~mL})$ and nitromethane $(25 \mathrm{~mL})$. The volume was reduced by half under reduced pressure. Then a solution of $3(1.92 \mathrm{~g}, 3.65 \mathrm{mM})$ in benzene/nitromethane ( $5 \mathrm{~mL}$ each) was added, followed by $\mathrm{Hg}(\mathrm{CN})_{2}(0.92 \mathrm{~g}, 3.65 \mathrm{mM})$ and $4 \mathrm{~A}^{0}$ molecular sieves as done in $\mathbf{8 a}$ to give a crude orange oil. Purification by silica gel chromatography (cyclohexane : EtOAc, 2 : 1) gave $0.25 \mathrm{~g}(16 \%)$ of $\mathbf{8 c}$ as a clear oil. FAB-MS $m / z 735(\mathrm{M}+\mathrm{Li})^{+} ;{ }^{1} \mathrm{HNMR}: \delta 7.60$ $(20 \mathrm{H}, \mathrm{m}, \mathrm{Ar} . H), 5.81,\left(1 \mathrm{H}, \mathrm{bs}, \mathrm{H}-1^{\prime}\right), 5.65\left(1 \mathrm{H}, \mathrm{dd}, J 2.0,0.9 \mathrm{~Hz}, \mathrm{H}-3^{\prime}\right), 5.50(1 \mathrm{H}, \mathrm{t}, J 1.3 \mathrm{~Hz}, \mathrm{H}-$ 2') 4.97 (1H, s, H-1), 4.83 (1H, m, H-5'a), 4.77 (1H, m, H-4'), 4.71 (1H, m, H-5'b), 4.30 (1H, m, $\mathrm{H}-4), 4.06(1 \mathrm{H}$, bs, H-2), $4.05(1 \mathrm{H}$, bs, H-3), $3.69(1 \mathrm{H}, \mathrm{m}, \mathrm{OH}), 3.62(1 \mathrm{H}, \mathrm{m}$, $\left.\mathrm{OCH} \mathrm{HCH}_{2} \mathrm{CH}_{2} \mathrm{C}_{6} \mathrm{H}_{5}\right), 3.40\left(1 \mathrm{H}, \mathrm{m},-\mathrm{OCH} \mathrm{HCH}_{2} \mathrm{CH}_{2} \mathrm{C}_{6} H_{5}\right), 3.25$ (1H, dd, $J$ 14.7, $\left.4.8 \mathrm{~Hz}, \mathrm{H}-5 \mathrm{a}\right)$, $2.97(1 \mathrm{H}, \mathrm{dd}, J 14.7,3.9 \mathrm{~Hz}, \mathrm{H}-5 \mathrm{~b}), 2.96(1 \mathrm{H}, \mathrm{m}, \mathrm{OH}), 2.64(2 \mathrm{H}, \mathrm{t}, J 7.9 \mathrm{~Hz},-$ $\mathrm{OCHHCH}_{2} \mathrm{CH}_{2} \mathrm{C}_{6} \mathrm{H}_{5}$ ), $1.88\left(2 \mathrm{H}, \mathrm{m}\right.$, $-\mathrm{OCHHCH}_{2} \mathrm{CH}_{2} \mathrm{C}_{6} \mathrm{H}_{5}$ ). (HRMS calcd for $\mathrm{C}_{40} \mathrm{H}_{40} \mathrm{O}_{11} \mathrm{~S}: \mathrm{m} / z$ $(\mathrm{M}+\mathrm{Na})^{+}$751.2183. Found $\left.m / z, 751.2195\right)$.

3'-Phenylpropyl-5-deoxy-5-S-( $\alpha$-D-arabinofuranosyl)-5-thio- $\alpha$-D-arabinofuranoside $\quad(9 \mathrm{c})$. To a solution of $8 \mathbf{c}(0.224 \mathrm{~g}, 0.30 \mathrm{mM})$ in dry $\mathrm{CHCl}_{3}(2.2 \mathrm{~mL})$ and dry $\mathrm{MeOH}(2.2 \mathrm{~mL})$ was added a solution of sodium metal $(20 \mathrm{mg})$ in dry $\mathrm{MeOH}(1.3 \mathrm{~mL})$ as mentioned in 9a. The residue was purified by silica gel chromatography $\left(\mathrm{CHCl}_{3}: \mathrm{MeOH}, 9: 1\right)$ to give $70 \mathrm{mg}(55 \%)$ of 9c as a clear oil. FAB-MS $m / z 417(\mathrm{M}+\mathrm{H})^{+} ;{ }^{1} \mathrm{H}$ NMR $\left(\mathrm{Me}_{2} \mathrm{SO}-d_{6}\right): \delta 7.22\left(5 \mathrm{H}, \mathrm{m},-\mathrm{C}_{6} H_{5}\right)$, $5.54(1 \mathrm{H}, 2-\mathrm{OH}), 5.40\left(1 \mathrm{H}, 2^{\prime}-\mathrm{OH}\right), 5.24\left(2 \mathrm{H}, 3-\mathrm{OH}, 3^{\prime}-\mathrm{OH}\right), 4.98(1 \mathrm{H}, \mathrm{d}, J 3.7 \mathrm{~Hz}, \mathrm{H}-1), 4.78$ $\left(1 \mathrm{H}, 5^{\prime}-\mathrm{OH}\right), 4.70\left(1 \mathrm{H}, \mathrm{d}, J 2.2 \mathrm{~Hz}, \mathrm{H}-1^{\prime}\right), 3.70\left(5 \mathrm{H}, \mathrm{m}, \mathrm{H}-2, \mathrm{H}-3, \mathrm{H}-3^{\prime}, \mathrm{H}-4^{\prime}\right.$, $\left.\mathrm{OCH} \mathrm{HCH}_{2} \mathrm{CH}_{2} \mathrm{C}_{6} \mathrm{H}_{5}\right), 3.45$ (2H, m, H-5'a,b), $3.30\left(1 \mathrm{H}, \mathrm{m},-\mathrm{OCH}^{\prime} \mathrm{CH}_{2} \mathrm{CH}_{2} \mathrm{C}_{6} \mathrm{H}_{5}\right), 2.92$ (1H, dd, $J$ 13.6, $3.9 \mathrm{~Hz}, \mathrm{H}-5 \mathrm{a}), 2.65(1 \mathrm{H}, \mathrm{dd}, J$ 13.6, $6.8 \mathrm{~Hz}, \mathrm{H}-5 \mathrm{~b}), 2.62(1 \mathrm{H}, \mathrm{t}, J 8.1 \mathrm{~Hz}$, $\mathrm{OCHHCH}_{2} \mathrm{CH}_{2} \mathrm{C}_{6} \mathrm{H}_{5}$ ), 1.79 (1H, m, -OCHHCH $\mathrm{CH}_{2} \mathrm{C}_{6} \mathrm{H}_{5}$ ). (HRMS calcd for $\mathrm{C}_{19} \mathrm{H}_{28} \mathrm{O}_{8} \mathrm{~S}: \mathrm{m} / z$ $(\mathrm{M}+\mathrm{Na})^{+}$439.1397. Found $\mathrm{m} / \mathrm{z}$ 439.1396). 
1-Thioacetyl-2,3,5-tri- $\boldsymbol{O}$-benzoyl-1-thio- $\boldsymbol{\alpha}$-D-arabinofuranoside (10). Compound $\mathbf{1}^{15}$ (500 mg, $1.05 \mathrm{mM})$ was dissolved in anhydrous $\mathrm{CH}_{2} \mathrm{Cl}_{2}(10 \mathrm{~mL})$, thiolacetic acid $(71 \mu \mathrm{L}, 0.99 \mathrm{mM})$ was added drop wise followed by $\mathrm{BF}_{3} \mathrm{OEt}_{2}(0.66 \mathrm{~mL}, 5.25 \mathrm{mM})$ at room temperature under the atmosphere of argon. The reaction mixture was stirred overnight at room temperature. Aq. $\mathrm{NaHCO}_{3}$ was added to it and reaction mixture was extracted twice with $\mathrm{CH}_{2} \mathrm{Cl}_{2}$. The organic layer was washed with water, dried over $\mathrm{Na}_{2} \mathrm{SO}_{4}$, filtered and concentrated under reduced pressure. Column chromatography (cyclohexane : EtOAc, $9.5: 0.5$ ) of the residue gave 10 (464 $\mathrm{mg}, 85 \%)$ as a colorless syrup. FAB-MS $\mathrm{m} / z 527(\mathrm{M}+\mathrm{Li})^{+} ;{ }^{1} \mathrm{HNMR} \delta 8.06(6 \mathrm{H}$, m, arom. $H$, ortho), 7.45 (9H, m, arom.H, meta, para), $6.32(1 \mathrm{H}, \mathrm{d}, J 0.6 \mathrm{~Hz}, \mathrm{H}-1), 5.70(1 \mathrm{H}$, broad s, H-2), $5.63(1 \mathrm{H}, \mathrm{m}, \mathrm{H}-3), 4.73$ (2H, m, H-5a,b), 4.62 (1H, m, H-4), 2.45 (3H, s, -SAc).

Octyl-2,3-di- $\boldsymbol{O}$-acetyl-5-deoxy-5-iodo- $\boldsymbol{\alpha}$-D-arabinofuranoside (12). A solution of $\mathrm{NaI}$ (840 $\mathrm{mg}, 5.6 \mathrm{mM})$ and $\mathbf{6 a}(400 \mathrm{mg}, 0.8 \mathrm{mM})$ in 2-butanone $(10 \mathrm{~mL})$ was refluxed for $5 \mathrm{~h}$. The reaction mixture was cooled to ambient temperature and filtered through a pad of celite. The filtrate was concentrated in reduced pressure and residue was partitioned between $\mathrm{CH}_{2} \mathrm{Cl}_{2}$ and water. The organic layer was washed with aq. $\mathrm{Na}_{2} \mathrm{~S}_{2} \mathrm{O}_{3}(0.1 \mathrm{~N})$, water, dried over $\mathrm{Na}_{2} \mathrm{SO}_{4}$, filtered and concentrated to give a clear oil. Column chromatography (cyclohexane : EtOAc, $9.5: 0.5$ ) of oil resulted in 12 (205 mg, 56\%). FAB-MS m/z $463(\mathrm{M}+\mathrm{Li})^{+}$; ${ }^{1} \mathrm{HNMR} \delta 5.09(1 \mathrm{H}, \mathrm{d}, J 1.3 \mathrm{~Hz}, \mathrm{H}-2)$, $5.03(1 \mathrm{H}, \mathrm{s}, \mathrm{H}-1), 4.87(1 \mathrm{H}, \mathrm{dd}, J 5.1,1 \mathrm{~Hz}, \mathrm{H}-3), 4.06(1 \mathrm{H}, \mathrm{m}, \mathrm{H}-4), 3.68(1 \mathrm{H}, \mathrm{m}$, $\left.\mathrm{OCH}\left(\mathrm{CH}_{2}\right)_{6} \mathrm{CH}_{3}\right), 3.45\left(3 \mathrm{H}, \mathrm{m}, \mathrm{H}-5 \mathrm{a}, \mathrm{b},-\mathrm{OCH}\left(\mathrm{CH}_{2}\right)_{6} \mathrm{CH}_{3}\right), 2.11(6 \mathrm{H}, \mathrm{s}, 2 \times \mathrm{OAc}), 1.57(2 \mathrm{H}$, m, $\left.-\mathrm{OCH}_{2} \mathrm{CH}_{2}\left(\mathrm{CH}_{2}\right)_{5} \mathrm{CH}_{3}\right), 1.27\left(10 \mathrm{H}, \mathrm{m},-\mathrm{OCH}_{2} \mathrm{CH}_{2}\left(\mathrm{CH}_{2}\right)_{5} \mathrm{CH}_{3}\right), 0.88(3 \mathrm{H}, \mathrm{t}, J 6.4 \mathrm{~Hz}$, $\left.\mathrm{OCH}_{2} \mathrm{CH}_{2}\left(\mathrm{CH}_{2}\right)_{5} \mathrm{CH}_{3}\right)$.

Octyl-2,3-di- $O$-acetyl-5- $S$-(2,3,4-tri- $O$-acetyl- $\beta$-D-arabinopyranosyl)-5-thio- $\alpha$-D-arabinofuranoside (13) and Octyl-2,3-di- $O$-acetyl-5-S-(2,3,5-tri- $O$-acetyl- $\beta$-D-arabinofuranosyl)-5thio- $\alpha$-D-arabinofuranoside (14). To a solution of $10(103 \mathrm{mg}, 0.2 \mathrm{mM})$ in anhydrous $\mathrm{MeOH}$ $(5 \mathrm{~mL})$, freshly prepared solution of $\mathrm{MeONa}(1 \mathrm{M}, 0.2 \mathrm{~mL})$ was added drop wise at $-78{ }^{0} \mathrm{C}$ under argon. After $1 \mathrm{~h}$ at room temperature, TLC confirmed the completion of the reaction. $\mathrm{MeOH}$ was evaporated off by gently passing the argon through the flask. The resulting residue $\mathbf{1 1}$ was dried overnight in vacuum pump.

The residue 11 and iodo sugar, $12(76 \mathrm{mg}, 0.16 \mathrm{mM})$ were dissolved separately in dry DMF ( $5 \mathrm{~mL}$ each) and mixed together slowly at room temperature under the atmosphere of argon. The reaction mixture was stirred for $48 \mathrm{~h}$ at room temperature. DMF was evaporated off under reduced pressure. The residue was taken in $\mathrm{CH}_{2} \mathrm{Cl}_{2}$, washed with aq. $\mathrm{NaHCO}_{3}$, water, dried over $\mathrm{Na}_{2} \mathrm{SO}_{4}$, filtered and concentrated to thick syrup. The syrup was acetylated with dry pyridine (3 $\mathrm{mL})$ and $\mathrm{Ac}_{2} \mathrm{O}(3 \mathrm{~mL})$ followed by usual work up and column chromatography (cyclohexane : EtOAc, $9: 1$ to $8: 2)$ gave 13 (30 mg, 25\%) and 14 (20 mg, 16\%).

13 : FAB-MS $m / z 627(\mathrm{M}+\mathrm{Li})^{+} ;{ }^{1} \mathrm{H}$ NMR $\delta 5.83\left(1 \mathrm{H}, \mathrm{d}, J 5.3 \mathrm{~Hz}, \mathrm{H}-1^{\prime}\right), 5.31\left(2 \mathrm{H}, \mathrm{m}, \mathrm{H}-2^{\prime}, \mathrm{H}^{-}\right.$ $\left.4^{\prime}\right), 5.22$ (1H, dd, $\left.J 10.3,3.3 \mathrm{~Hz}, \mathrm{H}-3^{\prime}\right), 5.03$ (1H, m, H-2), 4.97 (1H, s, H-1), 4.89 (1H, dd, J 5.5, 1.5H z, H-3), 4.30 (1H, dd, J 13.2, 1.5 Hz, H-5'a), 4.18 (1H, dd, J 11.6, 5.9 Hz, H-4), 3.67 (2H, m, H-5'b, -OCHH( $\left.\left(\mathrm{CH}_{2}\right)_{6} \mathrm{CH}_{3}\right), 3.42\left(1 \mathrm{H}, \mathrm{m},-\mathrm{OCH} H\left(\mathrm{CH}_{2}\right)_{6} \mathrm{CH}_{3}\right), 2.88(2 \mathrm{H}, \mathrm{d}, J 6.2 \mathrm{~Hz}, \mathrm{H}-5 \mathrm{a}, \mathrm{b})$, 2.133, 2.096, 2.093, 2.075, $2.023(3 \mathrm{H}, \mathrm{s}, 5 \times \mathrm{OAc}), 1.58\left(2 \mathrm{H}, \mathrm{m},-\mathrm{OCH}_{2} \mathrm{CH}_{2}\left(\mathrm{CH}_{2}\right)_{5} \mathrm{CH}_{3}\right), 1.27$ 
$\left(10 \mathrm{H}, \mathrm{m},-\mathrm{OCH}_{2} \mathrm{CH}_{2}\left(\mathrm{CH}_{2}\right)_{5} \mathrm{CH}_{3}\right), 0.88\left(3 \mathrm{H}, \mathrm{t}, J 6.4 \mathrm{~Hz},-\mathrm{OCH}_{2} \mathrm{CH}_{2}\left(\mathrm{CH}_{2}\right)_{5} \mathrm{CH}_{3}\right)$. (Found : C, 54.56, H, 7.17. $\mathrm{C}_{28} \mathrm{H}_{44} \mathrm{O}_{13} \mathrm{~S}$ required $\left.\mathrm{C}, 54.18, \mathrm{H}, 7.14\right)$.

14 : FAB-MS $m / z 627(\mathrm{M}+\mathrm{Li})^{+} ;{ }^{1} \mathrm{H}$ NMR $\delta 5.63\left(1 \mathrm{H}, \mathrm{d}, J 5.3 \mathrm{~Hz}, \mathrm{H}-1^{\prime}\right), 5.39(1 \mathrm{H}, \mathrm{dd}, J 5.0,1.1$ Hz, H-2'), 5.22 (1H, t, J 4.2 Hz, H-3'), 5.03 (1H, m, H-2), 4.98 (1H, s, H-1), 4.91 (1H, dd, J 7.3, $1.5 \mathrm{~Hz}, \mathrm{H}-3), 4.43$ (1H, dd, J 11.6, $5.3 \mathrm{~Hz}, \mathrm{H}-5$ 'a), 4.24 (2H, m, H-4', H-5'b), 4.10 (1H, m, H-4), $3.66\left(\mathrm{H}, \mathrm{m},-\mathrm{OCH}\left(\mathrm{CH}_{2}\right)_{6} \mathrm{CH}_{3}\right), 3.42\left(1 \mathrm{H}, \mathrm{m},-\mathrm{OCH} H\left(\mathrm{CH}_{2}\right)_{6} \mathrm{CH}_{3}\right), 2.99(2 \mathrm{H}, \mathrm{m}, \mathrm{H}-5 \mathrm{a}, \mathrm{b}), 2.13$, 2.12, 2.11, 2.10, $2.09(3 \mathrm{H}, \mathrm{s}, 5 \times \mathrm{OAc}), 1.59\left(2 \mathrm{H}, \mathrm{m},-\mathrm{OCH}_{2} \mathrm{CH}_{2}\left(\mathrm{CH}_{2}\right)_{5} \mathrm{CH}_{3}\right), 1.27(10 \mathrm{H}, \mathrm{m}$, $\left.\mathrm{OCH}_{2} \mathrm{CH}_{2}\left(\mathrm{CH}_{2}\right)_{5} \mathrm{CH}_{3}\right), 0.88\left(3 \mathrm{H}, \mathrm{t}, \mathrm{J} 6.4 \mathrm{~Hz},-\mathrm{OCH}_{2} \mathrm{CH}_{2}\left(\mathrm{CH}_{2}\right)_{5} \mathrm{CH}_{3}\right)$. (Found : C, 54.07, H, 6.75. $\mathrm{C}_{28} \mathrm{H}_{44} \mathrm{O}_{13} \mathrm{~S}$ required $\left.\mathrm{C}, 54.18, \mathrm{H}, 7.14\right)$.

1-Thioacetyl-2,3,5-tri- $\boldsymbol{O}$-benzyl-1-thio- $\boldsymbol{\alpha}$-D-arabinofuranoside (17). Compound $\mathbf{1 6}^{21}(1.79 \mathrm{~g}$, $4.12 \mathrm{mM}$ ) obtained from $\mathbf{1 5}^{18}$, was dissolved in anhydrous $\mathrm{CH}_{2} \mathrm{Cl}_{2}(20 \mathrm{ml})$, thiolacetic acid (392 $\mu \mathrm{L}, 5.44 \mathrm{mM})$ was added drop wise followed by $\mathrm{BF}_{3} \mathrm{OEt}_{2}(3.88 \mathrm{~m} \mathrm{~L}, 31 \mathrm{mM})$ at room temperature as done in 10. Column chromatography (cyclohexane : EtOAc, $9.5: 0.5$ ) of the residue gave 17 (340 mg, 29\%) and its $\beta$-anomer (200 mg, 17\%) as colorless syrups. FAB-MS $\mathrm{m} / \mathrm{z} 485(\mathrm{M}+\mathrm{Li})^{+} ;{ }^{1} \mathrm{HNMR} \delta 7.29(15 \mathrm{H}, \mathrm{m}$, arom. $H), 6.12(1 \mathrm{H}, \mathrm{s}, \mathrm{H}-1), 4.54\left(6 \mathrm{H}, \mathrm{m},-\mathrm{CH}_{2}\right.$ of Bn), 4.23 (1H, m, H-4), 4.13 (1H, t, J $1.5 \mathrm{~Hz}, \mathrm{H}-2), 3.98$ (1H, m, H-3), 3.59 (2H, m, H-5a,b), $2.36(3 \mathrm{H}, \mathrm{s},-\mathrm{SAc})$.

\section{Octyl-2,3-di- $O$-acetyl-5-S-(2,3,5-tri- $O$-benzyl- $\alpha$-D-arabinofuranosyl)-5-thio- $\alpha$-D-arabino-}

furanoside (19). To a solution of $17(230 \mathrm{mg}, 0.48 \mathrm{mM})$ in anhydrous $\mathrm{MeOH}(5 \mathrm{~mL})$, freshly prepared solution of $\mathrm{MeONa}(1 \mathrm{M}, 0.5 \mathrm{~mL})$ was added drop wise at $-78^{\circ} \mathrm{C}$ as reported in 13 and 14 to give thiosodium 18.

The residue 18 and tosyl derivative $6 \mathbf{a}(250 \mathrm{mg}, 0.5 \mathrm{mM})$ were dissolved separately in dry DMF ( $5 \mathrm{~mL}$ each) and mixed together slowly at room temperature as done in $\mathbf{1 3}$ and $\mathbf{1 4}$. Column chromatography (cyclohexane : EtOAc, $9.5: 0.5$ to $9: 1$ ) of the residue gave 19 (42 $\mathrm{mg}, 12 \%$ ). FAB-MS $m / z 771(\mathrm{M}+\mathrm{Li})^{+} ;{ }^{1} \mathrm{HNMR} \delta 7.28\left(15 \mathrm{H}, \mathrm{m}\right.$, arom.H), $5.50\left(1 \mathrm{H}, \mathrm{d}, J 2.2 \mathrm{~Hz}, \mathrm{H}-1^{\prime}\right), 5.06$ (2H, m, H-2, H-3), 4.96 (1H, s, H-1), 4.53 (6H, m, - $\mathrm{CH}_{2}$ of Bn), 4.31 (2H, m, H-4, H-4'), 3.99 $\left(2 \mathrm{H}, \quad \mathrm{m}, \quad \mathrm{H}-2^{\prime}, \quad \mathrm{H}-3^{\prime}\right), \quad 3.64\left(3 \mathrm{H}, \quad \mathrm{m}, \mathrm{H}-5^{\prime} \mathrm{a}, \mathrm{b}, \quad-\mathrm{OCH}\left(\mathrm{CH}_{2}\right)_{6} \mathrm{CH}_{3}\right), 3.39(1 \mathrm{H}, \mathrm{m}, \quad-$ $\left.\mathrm{OCH} H\left(\mathrm{CH}_{2}\right)_{6} \mathrm{CH}_{3}\right), 3.18(1 \mathrm{H}, \mathrm{dd}, J 14.1,4.5 \mathrm{~Hz}, \mathrm{H}-5 \mathrm{a}), 2.91(1 \mathrm{H}, \mathrm{dd}, J$ 14.1, $5.7 \mathrm{~Hz}, \mathrm{H}-5 \mathrm{~b})$, 2.06, 2.03, (3H, s, $2 \times \mathrm{OAc}), 1.57\left(2 \mathrm{H}, \mathrm{m}, \mathrm{OCH}_{2} \mathrm{CH}_{2}\left(\mathrm{CH}_{2}\right)_{5} \mathrm{CH}_{3}\right), 1.26(10 \mathrm{H}, \mathrm{m}$, $\left.\mathrm{OCH}_{2} \mathrm{CH}_{2}\left(\mathrm{CH}_{2}\right)_{5} \mathrm{CH}_{3}\right), 0.87\left(3 \mathrm{H}, \mathrm{t}, J 6.3 \mathrm{~Hz}, \mathrm{OCH}_{2} \mathrm{CH}_{2}\left(\mathrm{CH}_{2}\right)_{5} \mathrm{CH}_{3}\right)$. (Found : C, 66.91, H, 6.96. $\mathrm{C}_{43} \mathrm{H}_{56} \mathrm{O}_{10} \mathrm{~S}$ required $\left.\mathrm{C}, 66.51, \mathrm{H}, 7.37\right)$.

Methyl-2,3,5-tri- $\boldsymbol{O}$-methyl- $\boldsymbol{\alpha}$-D-arabinofuranoside (20). Compound $\mathbf{1 5}^{18}(1.73 \mathrm{~g}, 10.59 \mathrm{mM})$ was dissolved in dry DMF (50 mL) and $\mathrm{NaH}(60 \%$ dispersion in mineral oil, $2.12 \mathrm{~g}, 53 \mathrm{mM})$ was added. The reaction mixture was stirred at room temperature for $30 \mathrm{~min}$ followed by addition of iodomethane $(3.62 \mathrm{~mL}, 58.24 \mathrm{mM})$ dropwise at $0{ }^{\circ} \mathrm{C}$ and stirred for $4 \mathrm{~h}$ at room temperature. $\mathrm{MeOH}(20 \mathrm{~mL})$ was added, the solution was concentrated to dryness, the oil was redissolved in $\mathrm{CH}_{2} \mathrm{Cl}_{2}$ and organic layer was washed with water, brine, dried over $\mathrm{Na}_{2} \mathrm{SO}_{4}$, filtered and concentrated under reduced pressure. The residue was chromatographed (cyclohexane : EtOAc, 9 : 1) to yield $20(1.29 \mathrm{~g}, 60 \%)$. FAB-MS m/z $213(\mathrm{M}+\mathrm{Li})^{+} ;{ }^{1} \mathrm{HNMR} \delta 4.92(1 \mathrm{H}, \mathrm{s}, \mathrm{H}-1), 4.11$ 
(1H, m, H-4), 3.70 (1H, dd, J 2.2, 0.7 Hz, H-2), 3.59 (3H, m, H-3, H-5a,b), 3.422 , 3.419, 3.409, $3.402(3 \mathrm{H}, \mathrm{s}, 4 \mathrm{x}-\mathrm{OMe})$.

1-Thioacetyl-2,3,5-tri- $O$-methyl-1-thio- $\beta$-D-arabinofuranoside (21) and 1-Thioacetyl-2,3,5tri- $\boldsymbol{O}$-methyl-1-thio- $\boldsymbol{\alpha}$-D-arabinofuranoside (22). Compound 20 (1.28 g, $6.24 \mathrm{mM}$ ) was dissolved in anhydrous $\mathrm{CH}_{2} \mathrm{Cl}_{2}(15 \mathrm{ml})$, thiolacetic acid $(585 \mu \mathrm{L}, 8.11 \mathrm{mM})$ was added drop wise followed by $\mathrm{BF}_{3} \mathrm{OEt}_{2}(5.8 \mathrm{~mL}, 46.8 \mathrm{mM})$ as reported in $\mathbf{1 0}$ to give the thin oil which was column chromatographed (cyclohexane : EtOAc, 8.5 : 1.5) to give 21 (688 mg, 44\%) and 22 (279 mg, $18 \%)$.

21 : FAB-MS m/z $257(\mathrm{M}+\mathrm{Li})^{+} ;{ }^{1} \mathrm{HNMR} \delta 6.04(1 \mathrm{H}, \mathrm{d}, J 4.9 \mathrm{~Hz}, \mathrm{H}-1), 4.05$ (1H, m, H-4), 3.94 (1H, dd, J 4.9, 3.6 Hz, H-2), 3.71 (1H, t, J 3.8 Hz, H-3), 3.50 (2H, m, H-5a,b), 3.42, 3.41, 3.38 $(3 \mathrm{H}, \mathrm{s}, 3 \times \mathrm{OMe}), 2.37(3 \mathrm{H}, \mathrm{s}, \mathrm{SAc})$.

22 : FAB-MS m/z $257(\mathrm{M}+\mathrm{Li})^{+}$; ${ }^{1} \mathrm{HNMR} \delta 6.06(1 \mathrm{H}, \mathrm{d}, J 0.6 \mathrm{~Hz}, \mathrm{H}-1), 4.09$ (1H, dd, $J$ 10.1, 4.9 Hz, H-4), 3.89 (1H, t, J 1.3 Hz, H-2), 3.64 (1H, m, H-3), 3.53 (2H, m, H-5a,b), 3.43, 3.40, 3.39 $(3 \mathrm{H}, \mathrm{s}, 3 \times \mathrm{OMe}), 2.36(3 \mathrm{H}, \mathrm{s},-\mathrm{SAc})$.

Octyl-2,3-di- $O$-acetyl-5- $S$-(2,3,5-tri- $O$-methyl- $\alpha$-D-arabinofuranosyl)-5-thio- $\alpha$-D-arabinofuranoside (25). To a solution of $22(275 \mathrm{mg}, 1.1 \mathrm{mM})$ in anhydrous $\mathrm{MeOH}(5 \mathrm{~mL})$, freshly prepared solution of $\mathrm{MeONa}(1 \mathrm{M}, 1.1 \mathrm{~mL})$ was added drop wise at $-78{ }^{0} \mathrm{C}$ as reported in $\mathbf{1 3}$ and 14 to give thiosodium 24.

The residue 24 and tosyl derivative 6a $(550 \mathrm{mg}, 1.1 \mathrm{mM})$ were dissolved separately in dry DMF $(2 \mathrm{~mL}$ each) and mixed together slowly at room temperature as done in $\mathbf{1 3}$ and 14. Column chromatography (cyclohexane : EtOAc, $9: 1$ to $8.5: 1.5$ ) of the residue gave 25 (275 $\mathrm{mg}, 47 \%$ ). Similarly, compound 21 (100 mg, $0.4 \mathrm{mM}$ ) was reacted with $\mathrm{MeONa}$ to give 23 which on coupling with $\mathbf{6 a}(205 \mathrm{mg}, 0.4 \mathrm{mM})$ followed by column chromatography (cyclohexane : EtOAc, $9: 1$ to $8: 2)$ gave $25(50 \mathrm{mg}, 23 \%)$. FAB-MS $m / z, 542.8(\mathrm{M}+\mathrm{Li})^{+} ;{ }^{1} \mathrm{HNMR} \delta 5.43(1 \mathrm{H}, \mathrm{d}, J 1.9$ Hz, H-1'), 5.04 (2H, m, H-2, H-3), 4.96 (1H, s, H-1), 4.30 (1H, m, H-4), 4.20 (1H, m, H-4'), 3.62 (5H, m, H-5'a,b, H-3', H-2', -OCHH(CH$\left.)_{6} \mathrm{CH}_{3}\right), 3.42\left(1 \mathrm{H}, \mathrm{m},-\mathrm{OCH}\left(\mathrm{CH}_{2}\right)_{6} \mathrm{CH}_{3}\right), 3.412,3.411$, 3.399 (3H, s, $3 \times \mathrm{OMe}), 3.16$ (1H, dd, $J$ 14.1, $4.7 \mathrm{~Hz}, \mathrm{H}-5 \mathrm{a}), 2.88$ (1H, dd, J 14.1, $5.7 \mathrm{~Hz}, \mathrm{H}-5 \mathrm{~b})$, 2.09, $2.08(3 \mathrm{H}, \mathrm{s}, 2 \times \mathrm{OAc}), 1.57\left(2 \mathrm{H}, \mathrm{m},-\mathrm{OCH}_{2} \mathrm{CH}_{2}\left(\mathrm{CH}_{2}\right)_{5} \mathrm{CH}_{3}\right), 1.27(10 \mathrm{H}, \mathrm{m}$, $\left.\mathrm{OCH}_{2} \mathrm{CH}_{2}\left(\mathrm{CH}_{2}\right)_{5} \mathrm{CH}_{3}\right), 0.88\left(3 \mathrm{H}, \mathrm{t}, J 6.3 \mathrm{~Hz},-\mathrm{OCH}_{2} \mathrm{CH}_{2}\left(\mathrm{CH}_{2}\right)_{5} \mathrm{CH}_{3}\right)$. (Found : C, 56.01, H, 8.12. $\mathrm{C}_{25} \mathrm{H}_{44} \mathrm{O}_{10} \mathrm{~S}$ required $\left.\mathrm{C}, 55.95, \mathrm{H}, 8.26\right)$.

Octyl-5-S-(2,3,5-tri- $O$-methyl- $\alpha$-D-arabinofuranosyl)-5-thio- $\alpha$-D-arabinofuranoside. (26). Compound 25 (104 mg, $0.19 \mathrm{mM}$ ) was dissolved in dry $\mathrm{MeOH}$ ( 2mL) and methanolic ammonia $(7 \mathrm{~N}, 2 \mathrm{~mL})$ was added to it. The reaction mixture was stirred overnight at room temperature and solvents were evaporated under reduced pressure. Column chromatography (cyclohexane : EtOAc, $7: 3$ to $6: 4)$ of the residue gave $26(61 \mathrm{mg}, 70 \%)$. FAB-MS $m / z 459(\mathrm{M}+\mathrm{Li})^{+} ;{ }^{1} \mathrm{HNMR}$ $\delta 5.43\left(1 \mathrm{H}, \mathrm{d}, J 1.9 \mathrm{~Hz}, \mathrm{H}-1^{\prime}\right), 4.95$ (1H, s, H-1), 4.25 (2H, m, H-4, H-4'), 4.03 (1H, dd, J 8.5, 0.9 $\mathrm{Hz}, \mathrm{H}-2), 3.96$ (1H, m, H-3), 3.63 (5H, m, H-2', H-3', H-5'ab, - $\left.\mathrm{OCH}\left(\mathrm{CH}_{2}\right)_{6} \mathrm{CH}_{3}\right), 3.42(1 \mathrm{H}, \mathrm{m}$, $\left.-\mathrm{OCH} H\left(\mathrm{CH}_{2}\right)_{6} \mathrm{CH}_{3}\right), 3.41,3.40,3.39(3 \mathrm{H}, \mathrm{s}, 3 \times \mathrm{OMe}), 3.21(1 \mathrm{H}, \mathrm{dd}, J$ 14.6, $5.1 \mathrm{~Hz}, \mathrm{H}-5 \mathrm{a}), 2.99$ (1H, d, J 8.5 Hz, 2-OH), 2.89 (1H, dd, J 14.6, 3.4 Hz, H-5b), 2.83 (1H, d, J 9.9 Hz, 3-OH), 1.59 
$\left(2 \mathrm{H}, \mathrm{m},-\mathrm{OCH}_{2} \mathrm{CH}_{2}\left(\mathrm{CH}_{2}\right)_{5} \mathrm{CH}_{3}\right), 1.27\left(10 \mathrm{H}, \mathrm{m},-\mathrm{OCH}_{2} \mathrm{CH}_{2}\left(\mathrm{CH}_{2}\right)_{5} \mathrm{CH}_{3}\right), 0.88(3 \mathrm{H}, \mathrm{t}, J 6.3 \mathrm{~Hz}$, $\mathrm{OCH}_{2} \mathrm{CH}_{2}\left(\mathrm{CH}_{2}\right)_{5} \mathrm{CH}_{3}$ ). (Found : C, 55.67, H, 8.66. $\mathrm{C}_{21} \mathrm{H}_{40} \mathrm{O}_{8} \mathrm{~S}$ required C, 55.72, H, 8.90).

Methyl-2,3-di- $O$-methoxybenzyl-5- $O$ - $t$-butyldiphenylsily- $\alpha$-D-arabinofuranoside

(28).

Compound $27^{19}$ (5.22 g, $\left.12.97 \mathrm{mM}\right)$ was added to a suspension of $\mathrm{NaH}(60 \%$ dispersion in mineral oil, $1.24 \mathrm{~g}, 31.05 \mathrm{mM})$ in dry DMF $(40 \mathrm{~mL})$ drop wise at $0{ }^{0} \mathrm{C}$ followed by methoxybenzylchloride $(4.22 \mathrm{~mL}, 31.05 \mathrm{mM})$ and tetrabutylammonium bromide $(1 \mathrm{~g})$. The reaction mixture was stirred overnight at room temperature and was worked up as reported in 20. The residue was column chromatographed (cyclohexane : EtOAc, $1: 1)$ to yield 28 (3.7 g, $45 \%)$. FAB-MS $m / z 665(\mathrm{M}+\mathrm{Na})^{+}$; ${ }^{1} \mathrm{HNMR} \delta 7.51(10 \mathrm{H}, \mathrm{m}, \mathrm{PhH}$ of TBDPS), 7.18 (4H, m, methoxy $\mathrm{Bn} H$, ortho), $6.83\left(4 \mathrm{H}, \mathrm{m}\right.$, methoxyBnH, meta), $4.91(1 \mathrm{H}, \mathrm{s}, \mathrm{H}-1), 4.43\left(4 \mathrm{H}, \mathrm{m},-\mathrm{CH}_{2}\right.$ of methoxyBn), 4.11 (1H, m, H-4), 3.94 (2H, m, H-5a,b), 3.80, 3.77 (3H, s, $2 \times \mathrm{OMe}), 3.79$ (2H, m, $\mathrm{H}-2, \mathrm{H}-3), 3.37$ (3H, s, -OMe), 1.04 (9H, s, -CMe 3 ).

Methyl-2,3-di- $\boldsymbol{O}$-methoxybenzyl- $\boldsymbol{\alpha}$-D-arabinofuranoside. (29). Tetrabutylammonium fluoride $(8 \mathrm{~mL}, 7.95 \mathrm{mM})$ was added drop wise to a solution of $28(3.65 \mathrm{~g}, 5.68 \mathrm{mM})$ in freshly distilled THF $(50 \mathrm{~mL})$ at $0{ }^{\circ} \mathrm{C}$. The reaction was warmed upto room temperature and stirred for $3 \mathrm{~h}$. Brine water was added to the reaction mixture and extracted with EtOAc twice. The organic layer was dried over $\mathrm{Na}_{2} \mathrm{SO}_{4}$, filtered and concentrated under reduced pressure. The residue was passed through a column (cyclohexane : EtOAc, $8: 2$ to $6: 4$ ) to yield $29(1.63 \mathrm{~g}, 76 \%$ ). FAB-MS $\mathrm{m} / \mathrm{z}$ $411(\mathrm{M}+\mathrm{Li})^{+}$; ${ }^{1} \mathrm{HNMR} \delta 7.23$ (4H, m, methoxyBnH, ortho), 6.86 (4H, m, methoxyBn $H$, meta), $4.90(1 \mathrm{H}, \mathrm{s}, \mathrm{H}-1), 4.47\left(4 \mathrm{H}, \mathrm{m},-\mathrm{CH}_{2}\right.$ of methoxyBn), $4.10(1 \mathrm{H}, \mathrm{m}, \mathrm{H}-4), 3.93$ (2H, m, H-2, H-3), $3.82(1 \mathrm{H}, \mathrm{m}, \mathrm{H}-5 \mathrm{a}), 3.80,3.79$ (3H, s, $2 \times \mathrm{OMe}), 3.60(1 \mathrm{H}, \mathrm{m}, \mathrm{H}-5 \mathrm{~b}), 3.38$ (3H, s, $-\mathrm{OMe}), 1.86$ (1H, dd, J 7.9, $4.4 \mathrm{~Hz}, 5-\mathrm{OH})$.

Methyl-2,3-di- $O$-methoxybenzyl-5- $O$-benzyl- $\alpha$-D-arabinofuranoside. (30). Compound 29 (500 mg, $1.23 \mathrm{mM}$ ) was added to a suspension of $\mathrm{NaH}$ (60\% dispersion in mineral oil, $35.4 \mathrm{mg}$, $1.47 \mathrm{mM})$ in dry DMF $(10 \mathrm{~mL})$ drop wise at $0^{\circ} \mathrm{C}$ followed by addition of benzyl bromide $(204 \mu \mathrm{L}, 1.72 \mathrm{mM})$ as done in $\mathbf{2 0}$. The residue was column chromatographed (hexane : EtOAc, 8 : 2) to give 30 (600 mg, 98\%). FAB-MS m/z $501(\mathrm{M}+\mathrm{Li})^{+}$; ${ }^{1} \mathrm{HNMR} \delta 7.31(5 \mathrm{H}, \mathrm{m}, \mathrm{BnH}), 7.19$ (4H, m, methoxyBn $H$, ortho), 6.84 (4H, m, methoxyBn $H$, meta), 4.92 (1H, s, H-1), 4.55 (2H, m, $-\mathrm{CH}_{2}$ of $\left.\mathrm{Bn}\right), 4.43\left(4 \mathrm{H}, \mathrm{m},-\mathrm{CH}_{2}\right.$ of $\left.\mathrm{Bn}\right), 4.17(1 \mathrm{H}, \mathrm{m}, \mathrm{H}-4), 3.94(1 \mathrm{H}, \mathrm{dd}, J 2.8,1.1 \mathrm{~Hz}, \mathrm{H}-2)$, $3.85(1 \mathrm{H}, \mathrm{dd}, J 6.4,2.7 \mathrm{~Hz}, \mathrm{H}-3), 3.80,3.78$ (3H, s, $2 \times \mathrm{OMe}), 3.58$ (2H, m, H-5a,b), 3.38 (3H, s, -OMe).

Methyl-2,3-di- $\boldsymbol{O}$-acetyl-5- $\boldsymbol{O}$-benzyl- $\boldsymbol{\alpha}$-D-arabinofuranoside (32). Ceric ammonium nitrate (2.65 g, $4.84 \mathrm{mM})$ was added to $30(600 \mathrm{mg}, 1.21 \mathrm{mM})$ in $\mathrm{CH}_{3} \mathrm{CN}: \mathrm{H}_{2} \mathrm{O}(9: 1,20 \mathrm{~mL})$ at room temperature. After $4 \mathrm{~h}$, the reaction was diluted with aq. $\mathrm{NaHCO}_{3}$ and extracted with $\mathrm{CH}_{2} \mathrm{Cl}_{2}$ twice. The organic layer was washed with brine, dried $\left(\mathrm{Na}_{2} \mathrm{SO}_{4}\right)$, filtered and concentrated under reduced pressure to give 31. Acetylation of 31 with pyridine $(3 \mathrm{~mL})$ and $\mathrm{Ac}_{2} \mathrm{O}(3 \mathrm{~mL})$ followed by usual work up and column chromatography (cyclohexane : EtOAc, $9: 1$ to $8: 2$ ) yielded 32 (280 mg, 91\%). FAB-MS m/z $345(\mathrm{M}+\mathrm{Li})^{+} ;{ }^{1} \mathrm{HNMR} \delta 7.30(5 \mathrm{H}, \mathrm{m}, \mathrm{Bn} H), 5.08(1 \mathrm{H}, \mathrm{m}, \mathrm{H}-3)$, $5.04(1 \mathrm{H}, \mathrm{d}, J 1.3 \mathrm{~Hz}, \mathrm{H}-2), 4.94$ (1H, s, H-1), 4.61 (2H, m, $-\mathrm{CH}_{2}$ of Bn), 4.20 (1H, m, H-4), 3.77 
(1H, dd, J 10.8, 3.3 Hz, H-5a), 3.70 (1H, dd, J 10.8, 5.3 Hz, H-5b), 3.40 (3H, s, -OMe), 2.08, $2.04(3 \mathrm{H}, \mathrm{s}, 2 \times \mathrm{OAc})$.

1-Thioacetyl-2,3-di- $\boldsymbol{O}$-acetyl-5- $\boldsymbol{O}$-benzyl-1-thio- $\alpha$-D-arabinofuranoside (33). Compound 32 (260mg, 0.76mM) was dissolved in anhydrous $\mathrm{CH}_{2} \mathrm{Cl}_{2}(10 \mathrm{ml})$, thiolacetic acid $(75 \mu \mathrm{L}, 1.01 \mathrm{mM})$ was added drop wise followed by $\mathrm{BF}_{3} \mathrm{OEt}_{2}(0.71 \mathrm{~mL}, 5.7 \mathrm{mM})$ at room temperature as reported in 10. The residue was column chromatographed (cyclohexane : EtOAc, 9.1) to give 33 (191mg, $65 \%)$ as a colorless syrup. FAB-MS $m / z 389(\mathrm{M}+\mathrm{Li})^{+} ;{ }^{1} \mathrm{HNMR} \delta 7.30(5 \mathrm{H}, \mathrm{m}, \mathrm{Bn} H), 6.03(1 \mathrm{H}$, s, H-1), 5.22 (1H, t, J 1.43Hz, H-2), $5.16(1 \mathrm{H}, \mathrm{dd}, J 4.3,1 \mathrm{~Hz}, \mathrm{H}-3), 4.59$ (2H, m, - $\mathrm{CH}_{2}$ of Bn), $4.22(1 \mathrm{H}, \mathrm{m}, \mathrm{H}-4), 3.72(2 \mathrm{H}, \mathrm{d}, J 4.6 \mathrm{~Hz}, \mathrm{H}-5 \mathrm{a}, \mathrm{b}), 2.38$ (3H, s, -SAc), 2.11, 2.01 (3H, s, $2 \times$ OAc).

Octyl-2,3-di- $O$-acetyl-5-S-(2,3-di- $O$-acetyl-5- $O$-benzyl- $\beta$-D-arabinofuranosyl)-5-thio- $\alpha$-Darabinofuranoside (35) and Octyl-2,3-di- $\boldsymbol{O}$-acetyl-5-S-(2,3-di- $\boldsymbol{O}$-acetyl-5- $\boldsymbol{O}$-benzyl- $\boldsymbol{\alpha}$-Darabinofuranosyl)-5-thio- $\alpha$-D-arabinofuranoside (36). To a solution of $\mathbf{3 3}$ (176 mg, $0.46 \mathrm{mM})$ in anhydrous $\mathrm{MeOH}(5 \mathrm{~mL})$, freshly prepared solution of $\mathrm{MeONa}(1 \mathrm{M}, 0.46 \mathrm{~mL})$ was added drop wise at $-78{ }^{\circ} \mathrm{C}$ as reported in $\mathbf{1 3}$ and $\mathbf{1 4}$ to yield $\mathbf{3 4}$.

The residue 34 and tosyl derivative $6 \mathbf{a}(230 \mathrm{mg}, 0.46 \mathrm{mM})$ were dissolved separately in dry DMF ( $2 \mathrm{~mL}$ each) and mixed together slowly at room temperature as done in $\mathbf{1 3}$ and $\mathbf{1 4}$. The syrup was acetylated with pyridine $(3 \mathrm{ml})$ and $\mathrm{Ac}_{2} \mathrm{O}(3 \mathrm{~mL})$ followed by usual work up and column chromatography (cyclohexane : EtOAc, $9: 1$ to $8.5: 1.5)$ gave 35 (47 mg, 15\%) and 36 (36 mg, $12 \%)$.

35 : FAB-MS m/z $675(\mathrm{M}+\mathrm{Li})^{+} ;{ }^{1} \mathrm{HNMR} \delta 7.33(5 \mathrm{H}, \mathrm{m}$, arom. $H), 5.58\left(1 \mathrm{H}, \mathrm{d}, J 5.2 \mathrm{~Hz}, \mathrm{H}-1^{\prime}\right)$, $5.36\left(1 \mathrm{H}, \mathrm{dd}, J 5.0,3.6 \mathrm{~Hz}, \mathrm{H}-2^{\prime}\right), 5.24\left(1 \mathrm{H}, \mathrm{t}, J 4.0 \mathrm{~Hz}, \mathrm{H}-3{ }^{\prime}\right), 5.02(1 \mathrm{H}, \mathrm{d}, J 1.5 \mathrm{~Hz}, \mathrm{H}-2), 4.97$ $(1 \mathrm{H}, \mathrm{s}, \mathrm{H}-1), 4.91(1 \mathrm{H}, \mathrm{dd}, J 5.5,1.3 \mathrm{~Hz}, \mathrm{H}-3), 4.58\left(2 \mathrm{H}, \mathrm{m},-\mathrm{CH}_{2}\right.$ of Bn), $4.21(1 \mathrm{H}, \mathrm{dd}, J 10.5$, $5.8 \mathrm{~Hz}, \mathrm{H}-4), 4.09$ (1H, m, H-4'), 3.75 (2H, m, H-5'a,b), $3.67\left(1 \mathrm{H}, \mathrm{m},-\mathrm{OCHH}\left(\mathrm{CH}_{2}\right)_{6} \mathrm{CH}_{3}\right), 3.41$ $\left(1 \mathrm{H}, \mathrm{m},-\mathrm{OCH} H\left(\mathrm{CH}_{2}\right)_{6} \mathrm{CH}_{3}\right), 3.02(2 \mathrm{H}, \mathrm{m}, \mathrm{H}-5 \mathrm{a}, \mathrm{b}), 2.086,2.084,2.077,2.041(3 \mathrm{H}, \mathrm{s}, 4 \times \mathrm{OAc})$, $1.55\left(2 \mathrm{H}, \mathrm{m},-\mathrm{OCH}_{2} \mathrm{CH}_{2}\left(\mathrm{CH}_{2}\right)_{5} \mathrm{CH}_{3}\right), 1.26\left(10 \mathrm{H}, \mathrm{m},-\mathrm{OCH}_{2} \mathrm{CH}_{2}\left(\mathrm{CH}_{2}\right)_{5} \mathrm{CH}_{3}\right), 0.88(3 \mathrm{H}, \mathrm{t}, J 7.0$ $\mathrm{Hz},-\mathrm{OCH}_{2} \mathrm{CH}_{2}\left(\mathrm{CH}_{2}\right)_{5} \mathrm{CH}_{3}$ ). (Found : C, 59.30, H, 7.09. $\mathrm{C}_{33} \mathrm{H}_{48} \mathrm{O}_{12} \mathrm{~S}$ required C, 59.26, H, 7.23). 36 : FAB-MS m/z $675(\mathrm{M}+\mathrm{Li})^{+} ;{ }^{1} \mathrm{HNMR} \delta 7.33\left(5 \mathrm{H}, \mathrm{m}\right.$, arom.H), $5.47\left(1 \mathrm{H}, \mathrm{d}, J 0.6 \mathrm{~Hz}, \mathrm{H}-1^{\prime}\right)$, $5.16\left(1 \mathrm{H}, \mathrm{dd}, J 5.7,1.3 \mathrm{~Hz}, \mathrm{H}-3^{\prime}\right), 5.10\left(1 \mathrm{H}, \mathrm{t}, J 1.7 \mathrm{~Hz}, \mathrm{H}-2^{\prime}\right), 5.03$ (1H, broad s, H-3), 5.01 (1H, m, H-2), 4.97 (1H, s, H-1), $4.61\left(2 \mathrm{H}, \mathrm{m},-\mathrm{CH}_{2}\right.$ of Bn), $4.32(2 \mathrm{H}, \mathrm{m}, \mathrm{H}-4, \mathrm{H}-4$ ') 3.70 (3H, m, H5'a,b, - $\left.\mathrm{OCHH}\left(\mathrm{CH}_{2}\right)_{6} \mathrm{CH}_{3}\right), 3.40\left(1 \mathrm{H}, \mathrm{m},-\mathrm{OCH} H\left(\mathrm{CH}_{2}\right)_{6} \mathrm{CH}_{3}\right), 3.15(1 \mathrm{H}, \mathrm{dd}, J 14.1,4.5 \mathrm{~Hz}, \mathrm{H}-5 \mathrm{a})$, $2.95(1 \mathrm{H}, \mathrm{dd}, J 14.1,5.8 \mathrm{~Hz}, \mathrm{H}-5 \mathrm{~b}), 2.09,2.08,2.07,2.03(3 \mathrm{H}, \mathrm{s}, 4 \times \mathrm{OAc}), 1.55(2 \mathrm{H}, \mathrm{m}$, $\left.\mathrm{OCH}_{2} \mathrm{CH}_{2}\left(\mathrm{CH}_{2}\right)_{5} \mathrm{CH}_{3}\right), 1.26\left(10 \mathrm{H}, \mathrm{m},-\mathrm{OCH}_{2} \mathrm{CH}_{2}\left(\mathrm{CH}_{2}\right)_{5} \mathrm{CH}_{3}\right), 0.88(3 \mathrm{H}, \mathrm{t}, \quad J \quad 6.3 \mathrm{~Hz},-$ $\mathrm{OCH}_{2} \mathrm{CH}_{2}\left(\mathrm{CH}_{2}\right)_{5} \mathrm{CH}_{3}$ ). (Found : C, 59.27, H, 7.16. $\mathrm{C}_{33} \mathrm{H}_{48} \mathrm{O}_{12} \mathrm{~S}$ required $\mathrm{C}, 59.26, \mathrm{H}, 7.23$ ).

Methyl-5- $\boldsymbol{O}$-p-toluenesulfonyl- $\boldsymbol{\alpha}$-D-arabinofuranoside (37). To a dry pyridine (10 $\mathrm{mL})$ containing $15^{18}(1.5 \mathrm{~g}, 9.15 \mathrm{mM})$ was added dropwise a solution of $p$-toluenesulfonyl chloride $(1.92 \mathrm{~g}, 10.06 \mathrm{mM})$ in pyridine $(10 \mathrm{~mL})$ at $0{ }^{\circ} \mathrm{C}$ and the reaction mixture was stirred at room temperature overnight as mentioned earlier in $\mathbf{5}$ to give a crude pale oil. Purification by silica gel chromatography $\left(\mathrm{CH}_{2} \mathrm{Cl}_{2}: \mathrm{MeOH}, 9.5: 0.5\right.$ to $\left.9: 1\right)$ gave $37(1.72 \mathrm{~g}, 59 \%)$ as a clear oil. FABMS $m / z, 319(\mathrm{M}+\mathrm{H})^{+}$; ${ }^{1} \mathrm{HNMR} \delta 7.80(2 \mathrm{H}, \mathrm{d}, J 8.3 \mathrm{~Hz}$, arom.H, ortho), 7.35 (2H, d, $J 8.2$ 
$\mathrm{Hz}$, arom. $H$, meta), $4.91(1 \mathrm{H}, \mathrm{s}, \mathrm{H}-1), 4.22(3 \mathrm{H}, \mathrm{m}, \mathrm{H}-4, \mathrm{H}-5 \mathrm{a}, \mathrm{b}), 4.06(1 \mathrm{H}, \mathrm{dd}, J$ 7.0, 0.8 $\mathrm{Hz}, \quad \mathrm{H}-2), \quad 3.89, \quad(1 \mathrm{H}, \quad \mathrm{m}, \quad \mathrm{H}-3), \quad 3.37 \quad(3 \mathrm{H}, \quad \mathrm{s}, \quad-\mathrm{OMe}), \quad 2.60 \quad(1 \mathrm{H}, \quad \mathrm{d}, \quad J \quad 10.3$ $\mathrm{Hz}, 3-\mathrm{OH}), 2.45$ (3H, s, arom.Me), $2.12(1 \mathrm{H}, \mathrm{d}, J 7.5 \mathrm{~Hz}, 2-\mathrm{OH})$.

Methyl-2,3-di- $O$-acetyl-5-deoxy- $\alpha$-D-arabinofuranoside. (39). Lithium aluminium hydride $(160 \mathrm{mg}, 4 \mathrm{mM})$ in ether $(25 \mathrm{~mL})$ was added drop wise to $37(636 \mathrm{mg}, 2 \mathrm{mM})$ in ether $(75 \mathrm{~mL})$ over $30 \mathrm{~min}$ at room temperature. The reaction was stirred at room temperature overnight followed by addition of EtOAc and water. The suspension was filtered through celite and the resulting filtrate was evaporated to dryness by coevaporating with pyridine thrice to give $\mathbf{3 8}$. Acetylation of 38 with pyridine $(5 \mathrm{~mL})$ and $\mathrm{Ac}_{2} \mathrm{O}(5 \mathrm{~mL})$ followed by usual work up and column chromatography (cyclohexane : EtOAc, $8: 2$ ) yielded 39 (224 mg, 48\%). FAB-MS m/z 233 $(\mathrm{M}+\mathrm{H})^{+} ;{ }^{1} \mathrm{HNMR} \delta 5.04(1 \mathrm{H}, \mathrm{dd}, J$ 2.1, $0.4 \mathrm{~Hz}, \mathrm{H}-2), 4.85(1 \mathrm{H}, \mathrm{s}, \mathrm{H}-1), 4.77(1 \mathrm{H}, \mathrm{m}, \mathrm{H}-3), 4.13$ (1H, m, H-4), 3.38 (3H, s, -OMe), 2.103, 2.100 (3H, s, $2 \times$ OAc), 1.40 (3H, d, J 6.4 Hz, H-5).

1-Thioacetyl-2,3-di- $\boldsymbol{O}$-acetyl-5-deoxy-1-thio- $\boldsymbol{\alpha}$-D-arabinofuranoside (40). Compound 39 (202 $\mathrm{mg}, 0.87 \mathrm{mM})$ was dissolved in anhydrous $\mathrm{CH}_{2} \mathrm{Cl}_{2}(10 \mathrm{~mL})$, thiolacetic acid $(93 \mu \mathrm{L}, 1.25 \mathrm{mM})$ was added drop wise followed by $\mathrm{BF}_{3} \mathrm{OEt}_{2}(0.9 \mathrm{~mL}, 7.12 \mathrm{mM})$ at room temperature as done in 10. Column chromatography (cyclohexane : EtOAc, $9: 1)$ of the residue gave $\mathbf{4 0}$ (202 $\mathrm{mg}, 84 \%)$. FAB-MS m/z $283(\mathrm{M}+\mathrm{Li})^{+} ;{ }^{1} \mathrm{HNMR} \delta 5.97(1 \mathrm{H}, \mathrm{d}, J 0.5 \mathrm{~Hz}, \mathrm{H}-1), 5.23(1 \mathrm{H}, \mathrm{t}, J 1.5 \mathrm{~Hz}, \mathrm{H}-2)$, $3.83(1 \mathrm{H}, \mathrm{m}, \mathrm{H}-3), 4.14(1 \mathrm{H}, \mathrm{m}, \mathrm{H}-4), 2.39(3 \mathrm{H}, \mathrm{s},-\mathrm{SAc}), 2.12(6 \mathrm{H}, \mathrm{s}, 2 \times \mathrm{OAc}), 1.41(3 \mathrm{H}, \mathrm{d}, J$ $6.4 \mathrm{~Hz}, \mathrm{H}-5)$.

\section{Octyl-2,3-di- $O$-acetyl-5-S-(2,3-di- $O$-acetyl-5-deoxy- $\alpha-D$-arabinofuranosyl)-5-thio- $\alpha-D$-ara-} binofuranoside (42) and Octyl-2,3-di- $O$-acetyl-5-S-(2,3-di- $O$-acetyl-5-deoxy- $\beta$-D-arabinofuranosyl)-5-thio- $\alpha$-D-arabinofuranoside (43). To a solution of $40(238 \mathrm{mg}, 0.86 \mathrm{mM})$ in anhydrous $\mathrm{MeOH}(5 \mathrm{~mL})$, freshly prepared solution of $\mathrm{MeONa}(1 \mathrm{M}, 0.9 \mathrm{~mL})$ was added drop wise at $-78{ }^{\circ} \mathrm{C}$ as reported in $\mathbf{1 3}$ and $\mathbf{1 4}$ to yield $\mathbf{4 1}$.

The resulting residue $\mathbf{4 1}$ and tosyl derivative $\mathbf{6 a}(430 \mathrm{mg}, 0.86 \mathrm{mM})$ were dissolved separately in dry DMF (5 mL each) and mixed together slowly at room temperature as done in $\mathbf{1 3}$ and $\mathbf{1 4}$ to give crude syrup which was acetylated with pyridine $(3 \mathrm{ml})$ and $\mathrm{Ac}_{2} \mathrm{O}(3 \mathrm{~mL})$ followed by usual work up and column chromatography (cyclohexane : EtOAc, $9: 1$ to $8.5: 1.5$ ) gave 42 (45 mg, 9\%) and $\mathbf{4 3}$ (76 mg, 16\%).

42 : FAB-MS $m / z 569(\mathrm{M}+\mathrm{Li})^{+} ;{ }^{1} \mathrm{HNMR} \delta 5.38\left(1 \mathrm{H}, \mathrm{d}, J 0.7 \mathrm{~Hz}, \mathrm{H}-1^{\prime}\right), 5.08(1 \mathrm{H}, \mathrm{dd}, J 1.6,1.2$ $\left.\mathrm{Hz}, \mathrm{H}-2^{\prime}\right), 5.04(1 \mathrm{H}, \mathrm{s}, \mathrm{H}-2), 5.03(1 \mathrm{H}, \mathrm{m}, \mathrm{H}-3), 4.98(1 \mathrm{H}, \mathrm{s}, \mathrm{H}-1), 4.80\left(1 \mathrm{H}, \mathrm{m}, \mathrm{H}-3^{\prime}\right), 4.28(2 \mathrm{H}$, m, H-4, H-4') $3.66\left(1 \mathrm{H}, \mathrm{m},-\mathrm{OCH}\left(\mathrm{CH}_{2}\right)_{6} \mathrm{CH}_{3}\right), 3.42\left(1 \mathrm{H}, \mathrm{m},-\mathrm{OCH} H\left(\mathrm{CH}_{2}\right)_{6} \mathrm{CH}_{3}\right), 3.13(1 \mathrm{H}, \mathrm{dd}$, $J$ 14.0, 4.6 Hz, H-5a), 2.93 (1H, dd, $J$ 14.0, $5.7 \mathrm{~Hz}, \mathrm{H}-5 \mathrm{~b}), 2.105,2.104,(3 \mathrm{H}, \mathrm{s}, 2 \times \mathrm{OAc}), 2.09$ $(6 \mathrm{H}, \mathrm{s}, 2 \times \mathrm{OAc}), 1.55\left(2 \mathrm{H}, \mathrm{m},-\mathrm{OCH}_{2} \mathrm{CH}_{2}\left(\mathrm{CH}_{2}\right)_{5} \mathrm{CH}_{3}\right), 1.40\left(3 \mathrm{H}, \mathrm{d}, J 6.4 \mathrm{~Hz}, \mathrm{H}-5^{\prime}\right), 1.27(10 \mathrm{H}$, m, $\left.-\mathrm{OCH}_{2} \mathrm{CH}_{2}\left(\mathrm{CH}_{2}\right)_{5} \mathrm{CH}_{3}\right), 0.88\left(3 \mathrm{H}, \mathrm{t}, J 6.4 \mathrm{~Hz},-\mathrm{OCH}_{2} \mathrm{CH}_{2}\left(\mathrm{CH}_{2}\right)_{5} \mathrm{CH}_{3}\right.$ ). (Found : C, 55.69, H, 7.38. $\mathrm{C}_{26} \mathrm{H}_{42} \mathrm{O}_{11} \mathrm{~S}$ required $\left.\mathrm{C}, 55.50, \mathrm{H}, 7.52\right)$.

43 : FAB-MS $m / z 569(\mathrm{M}+\mathrm{Li})^{+} ;{ }^{1} \mathrm{HNMR} \delta 5.49\left(1 \mathrm{H}, \mathrm{d}, J 5.1 \mathrm{~Hz}, \mathrm{H}-1^{\prime}\right), 5.36(1 \mathrm{H}, \mathrm{dd}, J 5.1,3.5$ Hz, H-2'), $5.03(1 \mathrm{H}, \mathrm{d}, J 1.5 \mathrm{~Hz}, \mathrm{H}-2), 4.98(1 \mathrm{H}, \mathrm{s}, \mathrm{H}-1), 4.96\left(1 \mathrm{H}, \mathrm{m}, \mathrm{H}-3^{\prime}\right), 4.91(1 \mathrm{H}, \mathrm{m}, \mathrm{H}-3)$, $4.23(1 \mathrm{H}, \mathrm{m}, \mathrm{H}-4), 3.97\left(1 \mathrm{H}, \mathrm{m}, \mathrm{H}-4^{\prime}\right), 3.68\left(1 \mathrm{H}, \mathrm{m},-\mathrm{OCH}\left(\mathrm{CH}_{2}\right)_{6} \mathrm{CH}_{3}\right), 3.42(1 \mathrm{H}, \mathrm{m},-$ $\left.\mathrm{OCH} H\left(\mathrm{CH}_{2}\right)_{6} \mathrm{CH}_{3}\right), 3.04(2 \mathrm{H}, \mathrm{m}, \mathrm{H}-5 \mathrm{a}, \mathrm{b}), 2.121$ (3H, s, -OAc), $2.098(6 \mathrm{H}, \mathrm{s}, 2 \times \mathrm{OAc}), 2.096$ 
(3H, s, -OAc), $1.56\left(2 \mathrm{H}, \mathrm{m},-\mathrm{OCH}_{2} \mathrm{CH}_{2}\left(\mathrm{CH}_{2}\right)_{5} \mathrm{CH}_{3}\right), 1.46\left(3 \mathrm{H}, \mathrm{d}, J 6.6 \mathrm{~Hz}, \mathrm{H}-5^{\prime}\right), 1.27$ (10H, m, $\left.\mathrm{OCH}_{2} \mathrm{CH}_{2}\left(\mathrm{CH}_{2}\right)_{5} \mathrm{CH}_{3}\right), 0.88\left(3 \mathrm{H}, \mathrm{t}, \mathrm{J} 6.4 \mathrm{~Hz},-\mathrm{OCH}_{2} \mathrm{CH}_{2}\left(\mathrm{CH}_{2}\right)_{5} \mathrm{CH}_{3}\right)$. (Found : C, 55.51, H, 7.38. $\mathrm{C}_{26} \mathrm{H}_{42} \mathrm{O}_{11} \mathrm{~S}$ required $\mathrm{C}, 55.50, \mathrm{H}, 7.52$ ).

Methyl-2,3-di- $\boldsymbol{O}$-acetyl-5-deoxy-5-azido- $\boldsymbol{\alpha}$-D-arabinofuranoside. (45). $\mathrm{NaN}_{3}$ (572 $\mathrm{mg}, 8.8$ $\mathrm{mM})$ was added to a solution of $\mathbf{3 7}(700 \mathrm{mg}, 2.2 \mathrm{mM})$ in dry DMF $(10 \mathrm{~mL})$ at room temperature. The reaction was heated to $100{ }^{0} \mathrm{C}$ for $6 \mathrm{~h}$ followed by evaporation of DMF under reduced pressure. The residue was extracted with acetone : ether $(2: 1,10 \mathrm{~mL})$ thrice and filtered. The filtrate was evaporated to dryness under reduced pressure by coevaporating with pyridine thrice to give 44. Acetylation of $\mathbf{4 4}$ with pyridine $(5 \mathrm{~mL})$ and $\mathrm{Ac}_{2} \mathrm{O}(5 \mathrm{~mL})$ followed by usual work up and column chromatography (cyclohexane : EtOAc, $9: 1$ to $8: 2)$ yielded 45 (420 mg, 70\%). FAB-MS $m / z, 274(\mathrm{M}+\mathrm{H})^{+} ;{ }^{1} \mathrm{HNMR} \delta 5.08(1 \mathrm{H}, \mathrm{dd}, J$ 1.6, $0.4 \mathrm{~Hz}, \mathrm{H}-2), 4.97(1 \mathrm{H}, \mathrm{m}, \mathrm{H}-3), 4.95$ (1H, s, H-1), 4.17 (1H, m, H-4), 3.69 (1H, dd, J 13.2, 2.8 Hz, H-5a), 3.45 (1H, dd, J 13.2, 5.2Hz, H-5b), 3.41 (3H, s, -OMe), 2.12, 2.10 (3H, s, $2 \times \mathrm{OAc})$.

1-Thioacetyl-2,3-di- $\boldsymbol{O}$-acetyl-5-deoxy-5-azido- $\alpha$-D-arabinofuranoside (46). Compound 45 (420 mg, $1.53 \mathrm{mM})$ was dissolved in anhydrous $\mathrm{CH}_{2} \mathrm{Cl}_{2}(10 \mathrm{~mL})$, thiolacetic acid $(150 \mu \mathrm{L}, 2$ $\mathrm{mM})$ was added drop wise followed by $\mathrm{BF}_{3} \mathrm{OEt}_{2}(1.45 \mathrm{~mL}, 11.47 \mathrm{mM})$ at room temperature as done in 10. Column chromatography (cyclohexane : EtOAc, $9: 1$ to $8.5: 1.5$ ) of the residue gave 46 (257 mg, 53\%). FAB-MS m/z $318(\mathrm{M}+\mathrm{H})^{+} ;{ }^{1} \mathrm{HNMR} \delta 6.05(1 \mathrm{H}, \mathrm{d}, J 0.7 \mathrm{~Hz}, \mathrm{H}-1), 5.27(1 \mathrm{H}$, t, J 1.2 Hz, H-2), 5.04 (1H, m, H-3), 4.17 (1H, m, H-4), 3.66 (1H, dd, J 13.3, 3.5 Hz, H-5a), 3.46 $(1 \mathrm{H}, \mathrm{dd}, J 13.3,4.9 \mathrm{~Hz}, \mathrm{H}-5 \mathrm{~b}), 2.40$ (3H, s, -SAc), 2.15, 2.13 (3H, s, $2 \times \mathrm{OAc})$.

Octyl-2,3-di- $O$-acetyl-5- $S$-(2,3-di- $O$-acetyl-5-deoxy-5-azido- $\alpha$-D-arabinofuranosyl)-5-thio- $\alpha$ D-arabinofuranoside (48) and Octyl-2,3-di- $\boldsymbol{O}$-acetyl-5-S-(2,3-di- $\boldsymbol{O}$-acetyl-5-deoxy-5-azido- $\boldsymbol{\beta}$ D-arabinofuranosyl)-5-thio- $\alpha$-D-arabinofuranoside (49). To a solution of 46 (250 $\mathrm{mg}, 0.79$ $\mathrm{mM})$ in anhydrous $\mathrm{MeOH}(7 \mathrm{~mL})$, freshly prepared solution of $\mathrm{MeONa}(1 \mathrm{M}, .0 .8 \mathrm{~mL})$ was added drop wise at $-78^{\circ} \mathrm{C}$ as reported in $\mathbf{1 3}$ and $\mathbf{1 4}$ to yield thiosodium $\mathbf{4 7}$.

The resulting residue 47 and tosyl derivative $6 \mathbf{a}(395 \mathrm{mg}, 0.79 \mathrm{mM})$ were dissolved separately in dry DMF ( $5 \mathrm{~mL}$ each) and mixed together slowly at room temperature as done in $\mathbf{1 3}$ and $\mathbf{1 4}$ to give crude syrup which was acetylated with pyridine $(3 \mathrm{~mL})$ and $\mathrm{Ac}_{2} \mathrm{O}(3 \mathrm{~mL})$ followed by usual work up and column chromatography (cyclohexane : EtOAc, $9: 1$ to $8.5: 1.5$ ) gave 48 (24 mg, $9 \%$ ) and 49 (37 $\mathrm{mg}, 12 \%)$ beside recovering the tosyl derivative $6 \mathbf{a}(168 \mathrm{mg})$.

48 : FAB-MS $m / z 621\left(\mathrm{M}+\mathrm{NH}_{4}\right)^{+} ;{ }^{1} \mathrm{HNMR} \delta 5.48\left(1 \mathrm{H}, \mathrm{s}, \mathrm{H}-1^{\prime}\right), 5.14\left(1 \mathrm{H}, \mathrm{t}, J 1.4 \mathrm{~Hz}, \mathrm{H}-2^{\prime}\right), 5.05$ (1H, s, H-2), 5.02 (2H, m, H-3, H-3'), 4.99 (1H, s, H-1), 4.33 (2H, m, H-4, H-4'), 3.67 (2H, m, H-5'a, $\left.-\mathrm{OCH}\left(\mathrm{CH}_{2}\right)_{6} \mathrm{CH}_{3}\right), 3.45\left(2 \mathrm{H}, \mathrm{m}, \mathrm{H}-5\right.$ ' b, $\left.-\mathrm{OCH}\left(\mathrm{CH}_{2}\right)_{6} \mathrm{CH}_{3}\right), 3.16(1 \mathrm{H}, \mathrm{dd}, J$ 14.1, 4.4 Hz, H-5a), 2.94 (1H, dd, J 14.1, 5.6 Hz, H-5b), 2.12, 2.11, 2.10, 2.09 (3H, s, $4 \times$ OAc), 1.58 (2H, m, $\left.-\mathrm{OCH}_{2} \mathrm{CH}_{2}\left(\mathrm{CH}_{2}\right)_{5} \mathrm{CH}_{3}\right), 1.27\left(10 \mathrm{H}, \mathrm{m},-\mathrm{OCH}_{2} \mathrm{CH}_{2}\left(\mathrm{CH}_{2}\right)_{5} \mathrm{CH}_{3}\right), 0.88(3 \mathrm{H}, \mathrm{t}, J 6.4 \mathrm{~Hz}$, $\mathrm{OCH}_{2} \mathrm{CH}_{2}\left(\mathrm{CH}_{2}\right)_{5} \mathrm{CH}_{3}$ ). (Found : C, 51.50, H, 6.74, N, 7.10. $\mathrm{C}_{26} \mathrm{H}_{41} \mathrm{~N}_{3} \mathrm{O}_{11} \mathrm{~S}$ required $\mathrm{C}, 51.73, \mathrm{H}$, 6.84, N, 6.96).

49 : FAB-MS m/z $621\left(\mathrm{M}+\mathrm{NH}_{4}\right)^{+}$; ${ }^{1} \mathrm{HNMR} \delta 5.66\left(1 \mathrm{H}, \mathrm{d}, J 5.3 \mathrm{~Hz}, \mathrm{H}-1^{\prime}\right), 5.39(1 \mathrm{H}, \mathrm{dd}, J$ 5.2, $\left.4.0 \mathrm{~Hz}, \mathrm{H}-2^{\prime}\right), 5.14$ (1H, t, J 4.2 Hz, H-3'), 5.03 (1H, d, J $\left.1.5 \mathrm{~Hz}, \mathrm{H}-2\right), 4.98$ (1H, s, H-1), 4.91 $(1 \mathrm{H}, \mathrm{dd}, J$ 5.4, $1.3 \mathrm{~Hz}, \mathrm{H}-3), 4.21$ (1H, m, H-4), 4.03 (1H, m, H-4'), 3.68 (2H, m, H-5'a, - 
$\left.\mathrm{OCH}\left(\mathrm{CH}_{2}\right)_{6} \mathrm{CH}_{3}\right), 3.55\left(1 \mathrm{H}, \mathrm{dd}, J 13.0,3.9 \mathrm{~Hz}, \mathrm{H}-5\right.$ 'b), $3.42\left(1 \mathrm{H}, \mathrm{m},-\mathrm{OCH} H\left(\mathrm{CH}_{2}\right)_{6} \mathrm{CH}_{3}\right), 3.06$ $(2 \mathrm{H}, \mathrm{m}, \mathrm{H}-5 \mathrm{a}, \mathrm{b}), 2.13,2.10(3 \mathrm{H}, \mathrm{s}, 2 \times \mathrm{OAc}), 2.09(6 \mathrm{H}, \mathrm{s}, 2 \times \mathrm{OAc}), 1.58(2 \mathrm{H}, \mathrm{m},-$ $\left.\mathrm{OCH}_{2} \mathrm{CH}_{2}\left(\mathrm{CH}_{2}\right)_{5} \mathrm{CH}_{3}\right), 1.27\left(10 \mathrm{H}, \mathrm{m},-\mathrm{OCH}_{2} \mathrm{CH}_{2}\left(\mathrm{CH}_{2}\right)_{5} \mathrm{CH}_{3}\right), 0.88(3 \mathrm{H}, \mathrm{t}, J 6.4 \mathrm{~Hz}$, $\mathrm{OCH}_{2} \mathrm{CH}_{2}\left(\mathrm{CH}_{2}\right)_{5} \mathrm{CH}_{3}$ ). (HRMS calcd for $\mathrm{C}_{26} \mathrm{H}_{41} \mathrm{~N}_{3} \mathrm{O}_{11} \mathrm{~S}: \mathrm{m} / z$ ( $\left.\mathrm{M}+\mathrm{Na}\right)$ 626.2354. Found $\mathrm{m} / z$ 626.2332).

Octyl-5-S-(5-deoxy-5-azido- $\alpha$-D-arabinofuranosyl)-5-thio- $\alpha$-D-arabinofuranoside.

(50).

Compound 48 (24 mg, $0.04 \mathrm{mM}$ ) was dissolved in dry $\mathrm{MeOH}(2 \mathrm{~mL})$ and methanolic ammonia $(7 \mathrm{~N}, 2 \mathrm{~mL})$ was added to it as done in $\mathbf{2 6}$ to give thick syrup which was column chromatographed $\left(\mathrm{CH}_{2} \mathrm{Cl}_{2}: \mathrm{MeOH}, 97: 3\right)$ to give 50 (15 mg, 88\%). FAB-MS m/z $442(\mathrm{M}+\mathrm{Li})^{+}$; ${ }^{1} \mathrm{HNMR}\left(\mathrm{DMSO}-d_{6}\right) \delta 5.68\left(1 \mathrm{H}, \mathrm{d}, J 5.3 \mathrm{~Hz}, 2^{\prime}-\mathrm{OH}\right), 5.46\left(1 \mathrm{H}, \mathrm{d}, J 5.2 \mathrm{~Hz}, 3^{\prime}-\mathrm{OH}\right), 5.38(1 \mathrm{H}, \mathrm{d}$, $J 5.1 \mathrm{~Hz}, 2-\mathrm{OH}), 5.20(1 \mathrm{H}, \mathrm{d}, J 5.5 \mathrm{~Hz}, 3-\mathrm{OH}), 5.03\left(1 \mathrm{H}, \mathrm{d}, J 3.9 \mathrm{~Hz}, \mathrm{H}-1^{\prime}\right), 4.68(1 \mathrm{H}, \mathrm{d}, J 2.2$ Hz, H-1), 3.88 (2H, m, H-4, H-4'), 3.74 (2H, dd, J 4.5, 1.6 Hz, H-2, H-2'), 3.61 (4H, m, H-3, H3', H-5'a, -OCHH(CH$\left.)_{6} \mathrm{CH}_{3}\right), 3.39\left(1 \mathrm{H}, \mathrm{m}, \mathrm{H}-5\right.$ 'b), $3.31\left(1 \mathrm{H}, \mathrm{m},-\mathrm{OCH} H\left(\mathrm{CH}_{2}\right)_{6} \mathrm{CH}_{3}\right), 2.92(1 \mathrm{H}$, dd, J 13.5, $3.9 \mathrm{~Hz}, \mathrm{H}-5 \mathrm{a}), 2.68(1 \mathrm{H}, \mathrm{dd}, J$ 13.5, $6.9 \mathrm{~Hz}, \mathrm{H}-5 \mathrm{~b}), 1.49\left(2 \mathrm{H}, \mathrm{m},-\mathrm{OCH}_{2} \mathrm{CH}_{2-}\right.$ $\left.\left(\mathrm{CH}_{2}\right)_{5} \mathrm{CH}_{3}\right), 1.25\left(10 \mathrm{H}, \mathrm{m},-\mathrm{OCH}_{2} \mathrm{CH}_{2}\left(\mathrm{CH}_{2}\right)_{5} \mathrm{CH}_{3}\right), 0.85\left(3 \mathrm{H}, \mathrm{t}, \quad J \quad 6.8 \mathrm{~Hz},-\mathrm{OCH}_{2} \mathrm{CH}_{2-}\right.$ $\left(\mathrm{CH}_{2}\right)_{5} \mathrm{CH}_{3}$ ). (Found : C, 49.44, H, 7.52, N, 9.86. $\mathrm{C}_{18} \mathrm{H}_{33} \mathrm{~N}_{3} \mathrm{O}_{7} \mathrm{~S}$ required $\mathrm{C}, 49.64, \mathrm{H}, 7.64, \mathrm{~N}$, 9.65).

Octyl-5-S-(5-deoxy-5-azido- $\beta$-D-arabinofuranosyl)-5-thio- $\alpha$-D-arabinofuranoside.

(51). Compound 49 (24 mg, $0.04 \mathrm{mM}$ ) was dissolved in dry $\mathrm{MeOH}(2 \mathrm{~mL})$ and methanolic ammonia $(7 \mathrm{~N}, 2 \mathrm{~mL})$ was added to it as done in $\mathbf{2 6}$ to give crude syrup which was column chromatographed $\left(\mathrm{CH}_{2} \mathrm{Cl}_{2}: \mathrm{MeOH}, 97: 3\right)$ to give 51 (16 mg, 94\%). FAB-MS m/z $442(\mathrm{M}+\mathrm{Li})^{+} ;{ }^{1} \mathrm{HNMR}$ (DMSO-d $)_{6} \delta 5.63\left(1 \mathrm{H}, \mathrm{d}, J 4.8 \mathrm{~Hz}, 2^{\prime}-\mathrm{OH}\right), 5.43\left(1 \mathrm{H}, \mathrm{d}, J 4.4 \mathrm{~Hz}, 3^{\prime}-\mathrm{OH}\right), 5.39(1 \mathrm{H}, \mathrm{d}, J 4.9 \mathrm{~Hz}$, H-1'), 5.36 (1H, d, J 5.2 Hz, 2-OH), $5.22\left(1 \mathrm{H}, \mathrm{d}, J 5.5 \mathrm{~Hz}, 3^{\prime}-\mathrm{OH}\right), 4.67$ (1H, d, J 1.8 Hz, H-1), 4.01 (1H, t, J 4.4 Hz, H-2'), 3.81 (3H, m, H-4, H-3', H-4'), 3.73 (1H, dd, J 4.6, 1.9 Hz, H-2), 3.52 (3H, m, H-3, H-5'a, -OCHH(CH$\left.)_{6} \mathrm{CH}_{3}\right), 3.32\left(2 \mathrm{H}, \mathrm{m}, \mathrm{H}-5\right.$ 'b, - $\left.\mathrm{OCH} H\left(\mathrm{CH}_{2}\right)_{6} \mathrm{CH}_{3}\right), 2.87(1 \mathrm{H}, \mathrm{dd}$, $J$ 13.5, 3.7 Hz, H-5a), $2.73(1 \mathrm{H}, \mathrm{dd}, J 13.5,7.8 \mathrm{~Hz}, \mathrm{H}-5 \mathrm{~b}), 1.49\left(2 \mathrm{H}, \mathrm{m},-\mathrm{OCH}_{2} \mathrm{CH}_{2}\left(\mathrm{CH}_{2}\right)_{5} \mathrm{CH}_{3}\right)$, $1.25\left(10 \mathrm{H}, \mathrm{m},-\mathrm{OCH}_{2} \mathrm{CH}_{2}\left(\mathrm{CH}_{2}\right)_{5} \mathrm{CH}_{3}\right), 0.86\left(3 \mathrm{H}, \mathrm{t}, J 6.8 \mathrm{~Hz},-\mathrm{OCH}_{2} \mathrm{CH}_{2}\left(\mathrm{CH}_{2}\right)_{5} \mathrm{CH}_{3}\right)$. (HRMS calcd for $\mathrm{C}_{18} \mathrm{H}_{33} \mathrm{~N}_{3} \mathrm{O}_{7} \mathrm{~S}: \mathrm{m} / z(\mathrm{M}+\mathrm{Na}) 458.1931$. Found $\mathrm{m} / z$ 458.1944).

Octyl-5- $S$-(5-deoxy-5-acetamido- $\beta$-D-arabinofuranosyl)-5-thio- $\alpha$-D-arabinofuranoside. (52). $\mathrm{Ph}_{3} \mathrm{P}(11 \mathrm{mg}, 0.04 \mathrm{mM})$ and water $(4.6 \mu \mathrm{L}, 0.25 \mathrm{mM})$ were added to compound 49 (10 mg. 0.016 $\mathrm{mM})$ in freshly distilled THF $(1 \mathrm{~mL})$ and stirred overnight at $65{ }^{0} \mathrm{C}$. The TLC showed the mixture of products of close mobility in reaction mixture. Solvents were evaporated off under vacuum to dryness and the residue was deacetylated with methanolic ammonia $(7 \mathrm{~N}, 2 \mathrm{~mL})$ in dry $\mathrm{MeOH}(2 \mathrm{~mL})$. Solvents were evaporated under reduced pressure. The residue was passed through short column $\left(\mathrm{CH}_{2} \mathrm{Cl}_{2}: \mathrm{MeOH}, 99: 1\right)$ to give 52 (5 mg, 42\%). FAB-MS m/z 452 $(\mathrm{M}+\mathrm{H})^{+} ;{ }^{1} \mathrm{HNMR}\left(\mathrm{DMSO}-d_{6}\right) \delta 5.53\left(1 \mathrm{H}, \mathrm{d}, J 4.8 \mathrm{~Hz}, 2^{\prime}-\mathrm{OH}\right), 5.38(1 \mathrm{H}, \mathrm{d}, J 4.9 \mathrm{~Hz}, 2-\mathrm{OH})$, $5.29\left(1 \mathrm{H}, \mathrm{d}, J 4.7 \mathrm{~Hz}, \mathrm{H}-1^{\prime}\right), 5.28\left(1 \mathrm{H}, \mathrm{d}, J 3.5 \mathrm{~Hz}, 3^{\prime}-\mathrm{OH}\right), 5.23(1 \mathrm{H}, \mathrm{d}, J 5.3 \mathrm{~Hz}, 3-\mathrm{OH}), 4.67$ $(1 \mathrm{H}, \mathrm{d}, J 1.9 \mathrm{~Hz}, \mathrm{H}-1), 3.96\left(1 \mathrm{H}, \mathrm{t}, J 4.0 \mathrm{~Hz}, \mathrm{H}-2^{\prime}\right), 3.83\left(1 \mathrm{H}, \mathrm{m}, \mathrm{H}-4^{\prime}\right), 3.78$ (1H, t, J 4.1 Hz, H3'), $3.74(1 \mathrm{H}, \mathrm{dd}, J 4.5,2.1 \mathrm{~Hz}, \mathrm{H}-2), 3.64\left(1 \mathrm{H}, \mathrm{m}, \mathrm{H}-4^{\prime}\right), 3.57$ (1H, dd, J 6.9, $\left.4.2 \mathrm{~Hz}, \mathrm{H}-3\right), 3.53$ $\left(1 \mathrm{H}, \mathrm{m},-\mathrm{OCH}\left(\mathrm{CH}_{2}\right)_{6} \mathrm{CH}_{3}\right), 3.32\left(2 \mathrm{H}, \mathrm{m}, \mathrm{H}-5\right.$ 'a, $\left.-\mathrm{OCH}\left(\mathrm{CH}_{2}\right)_{6} \mathrm{CH}_{3}\right), 3.16(1 \mathrm{H}, \mathrm{dd}, J$ 13.4, 6.9 
Hz, H-5'b), 2.87 (1H, dd, J 13.6, 3.8 Hz, H-5a), 2.73 (1H, dd, J 13.6, 7.8 Hz, H-5b), 1.48 (2H, m, $\left.-\mathrm{OCH}_{2} \mathrm{CH}_{2}\left(\mathrm{CH}_{2}\right)_{5} \mathrm{CH}_{3}\right), 1.25\left(10 \mathrm{H}, \mathrm{m},-\mathrm{OCH}_{2} \mathrm{CH}_{2}\left(\mathrm{CH}_{2}\right)_{5} \mathrm{CH}_{3}\right), 0.85(3 \mathrm{H}, \mathrm{t}, J 6.8 \mathrm{~Hz}$, $\mathrm{OCH}_{2} \mathrm{CH}_{2}\left(\mathrm{CH}_{2}\right)_{5} \mathrm{CH}_{3}$ ). (HRMS calcd for $\mathrm{C}_{20} \mathrm{H}_{37} \mathrm{NO}_{8} \mathrm{~S}: \mathrm{m} / \mathrm{z}(\mathrm{M}+\mathrm{Na})^{+}$474.2132. Found $\mathrm{m} / \mathrm{z}$ 474.2128).

\section{References}

1. (a) World Health Organization, in Anti-tuberculosis Drug Resistance in the World. The WHO / IUATLD global project on anti-tuberculosis drug resistance surveillance, 1997. (b) Cohn, D. L.; Bustreo, F.; Raviglione, M.C. Clin. Infect. Dis. 1997, 24, S121. http://dx.doi.org/10.1093/clinids/24.Supplement_1.S121, PMid:8994791. (c) Douglas, J.G.; McLeod, M.-J. Clin. Pharmacokinetics 1999, 37, 127. http://dx.doi.org/10.2165/00003088199937020-00003, PMid:10496301. (d) Basso, L. A.; Blanchard, J. S. in Resolving the Antibiotic Paradox; Rosen and Mobashery, Eds. Resistance to Antitubercular Drugs. Plenum Publishers, New York, 1998, p 115. (e) Bastian, I.; Colebunders, R. Drugs 1999, 58 633. http://dx.doi.org/10.2165/00003495-199958040-00005, PMid:10551435. (f) Bradford, W. Z.; Daley, C. L. Infect. Dis. Clin. North Am. 1998, 12, 157. http://dx.doi.org/10.1016/S0891-5520(05)70415-3. (g) Rattan, A.; Kalia, A.; Ahmad, N. Emerging Infect. Dis. 1998, 4, 195. http://dx.doi.org/10.3201/eid0402.980207, PMid:9621190 PMCid:2640153. (h) Butler D. Nature 2000, 406, 670. http://dx.doi.org/10.1038/35021291, PMid:10963570.

2. (a) Bodiang, C. K. Scot. Med. J. 2000, 45, 25. (b) Van Scoy, R. E.; Wilkowske, C. J. Mayo Clin. Proc. 1999, 74, 1038. (c) Sung, S.-W.; Kang, C. H.; Kim, Y. T.; Han, S. K.; Shim, Y.S.; Kim, J. H. Eur. J. Cardio-Thoracic Surg. 1999, 16, 187. http://dx.doi.org/10.1016/S1010-7940(99)00158-X.

3. (a) Ravilione, M. C. Scot. Med. J. 2000, 45, 52. (b) Long, R. CMAJ 2000, 163, 425. PMid:10976260 PMCid:80378. (c) Walsh, C. Nature 2000, 406, 775. http://dx.doi.org/10.1038/35021219, PMid:10963607

4. NIAID, Web Site, http://www.niaid.nih.gov/factsheets/tb.htm, http://www.niaid.nih.gov/factsheets/tbrsch.htm, http://www.who.int/gtb/publications/factsheet/index.htm

5. (a) Chatterjee, D. Curr. Opin. Chem. Biol. 1997, 1, 579. http://dx.doi.org/10.1016/S13675931(97)80055-5. (b) Daffe, M.; Draper, P. Adv. Microb. Physiol. 1998, 39, 131. http://dx.doi.org/10.1016/S0065-2911(08)60016-8.

6. (a) Wolucka, B. A.; McNeil, M. R.; deHoffman, E.; Chojnacki, T.; Brennan, P. J. J. Biol. Chem. 1994, 269, 23328. (b) Lee, R. E.; Mikusova, K.; Brennan, P. J.; Besra, G. S. J. Am. Chem. Soc. 1995, 117, 11829. http://dx.doi.org/10.1021/ja00153a002. (c) Scherman, M. S.; Kalbe-Bournonville, L.; Bush, D.; Xin, Y.; Deng, L.; McNeil, M. J. Biol. Chem. 1996, 271, 29652. http://dx.doi.org/10.1074/jbc.271.47.29652. 
7. (a) Winder, F. G. In The Biology of the Mycobacteria, Vol. 1; Ratledge, C.; Standford, J., Eds. Academic Press : London, 1982; pp 417-521. (b) Mikusova, K.; Slayden, R. A.; Besra, G. S.; Brennan, P. J. Antimicrob. Agents Chemother. 1995, 39, 2484. http://dx.doi.org/10.1128/AAC.39.11.2484.

8. Takayama, K.; Kilburam, J. O. Antimicrob. Ag. Chemother. 1989, 33, 1493. http://dx.doi.org/10.1128/AAC.33.9.1493, PMid:2817850 PMCid:172689.

9. (a) Lee, R. E.; Mikusova, K.; Brennan, P. J.; Besra, G. S. J. Am. Chem. Soc. 1995, 117, 1182. http://dx.doi.org/10.1021/ja00153a002. (b) Lee, R. E.; Brennan, P. J.; Besra, G. S. Glycobiology 1997, 7, 1121. http://dx.doi.org/10.1093/glycob/7.8.1121. PMid:9455913. (c) Pathak, A. K.; Pathak, V.; Bansal, N.; Maddry, J. A.; Reynolds, R. C. Tetrahedron Lett. 2001, 42, 979. http://dx.doi.org/10.1016/S0040-4039(00)02161-4.

10. (a) Maddry, J. A.; Bansal, N.; Bermudez, L. E.; Comber, R. N.; Orme, I. A.; Suling, W. J.; Reynolds, R. C. Bioorg. Med. Chem.Lett. 1998, 8, 237. http://dx.doi.org/10.1016/S0960894X(98)00017-1. (b) Brown, J. R.; Smith, T. K.; Ferguson, M. A. J.; Field, R. A. Bioorg. Med. Chem.Lett. 1998, 8, 2051. http://dx.doi.org/10.1016/S0960-894X(98)00359-X.

11. Blanc-Muesser, M.; Defaye, J.; Driguez, H. Tetrahedron Lett. 1976, 4307-4310. http://dx.doi.org/10.1016/0040-4039(76)80102-5.

12. Hutson, D. H.; J. Chem. Soc. C. 1967, 442. http://dx.doi.org/10.1039/j39670000442.

13. (a) Pathak, A. K.; Pathak, V.; Khare, N. K.; Maddry, J. A.; Reynolds, R. C. Carbohydr. Lett. 2001, 4(2), 117-122. PMid:11506156. (b) Srivastava, J.; Khare, A.; Khare. N. K. Arkivoc 2009, (vii), 180.

14. Luo, S; Tripathi, A; Zulueta, M. M. L; Hung, S. Carbohydr. Res. 2012, 352, 197-201. http://dx.doi.org/10.1016/j.carres.2012.01.022, PMid: 22370177.

15. (a) Ness, R. K.; Fletcher, H. G. J. Am. Chem. Soc. 1958, 80, 2007. http://dx.doi.org/10.1021/ja01541a058. (b) Srivastava, J.; Khare, A.; Khare, N. K. Carbohydr. Res. 2008, 343, 2822. http://dx.doi.org/10.1016/j.carres.2008.08.006; PMid:18804201.

16. Lee, H. C.; Kumar, P.; Wiebe, L. I.; McDonald, R.; Mercer, J. R.; Ohkura, K.; Seki, Koh$\begin{array}{lllll}\text { Ichi. } & \text { Nucleosides } & \text { Nucleotides } & 1999, & 18,\end{array}$ http://dx.doi.org/10.1080/07328319908044860

17. (a) Pathak, A. K.; El-Kattan, Y. A.; Bansal, N.; Maddry, J. A.; Reynolds, R. C. Tetrahedron Lett. 1998, 39, 1497. http://dx.doi.org/10.1016/j.bmc.2008.11.027. PMid:19056279 PMCid:2707774. (b) Pathak, A. K.; Pathak, V.; Suling, W. J.; Riordan, J. R.; Gurcha, S. S.; Besra, G. S.; Reynolds, R. C. Bioorg. Med. Chem. 2009, 17, 872. http://dx.doi.org/10.1016/j.bmc.2008.11.027, PMid:19056279 PMCid:2707774.

18. Callam, C.S.; Gadikota, R. R.; Lowary, T. L.; Carbohydr. Res. 2001, 330, 267. http://dx.doi.org/10.1016/S0008-6215(00)00277-9.

19. D’Souza, F. W.; Ayers, J. D.; McCarren, P. R.; Lowary, T. D. J. Am. Chem. Soc. 2000, 122, 1251. http://dx.doi.org/10.1021/ja9935431. 
20. (a) Pathak, A. K.; Pathak, V.; Maddry, J. A.; Suling, W. J.; Gurcha, S. S.; Besra, G. S.; Reynolds, R. C. Bioorg. Med. Chem. 2001, 9, 3145. http://dx.doi.org/10.1016/S09680896(01)00180-8. (b) Khare, N. K.; Reynolds, R. C.; Maddry, J. A. Ind. J. Chem., Sec. B, 2008, 47B, 1748.

21. Dhawan, S. N.; Goux, W. J. Carbohydr. Res. 1988, 183, 47. http://dx.doi.org/10.1016/00086215(88)80044-2. 